\title{
THE COMPLEX LORENTZIAN LEECH LATTICE AND THE BIMONSTER (II)
}

\author{
TATHAGATA BASAK
}

Abstract. Let $D$ be the incidence graph of the projective plane over $\mathbb{F}_{3}$. The Artin group of the graph $D$ maps onto the bimonster and a complex hyperbolic reflection group $\Gamma$ acting on 13 dimensional complex hyperbolic space $Y$. The generators of the Artin group are mapped to elements of order 2 (resp. 3) in the bimonster (resp. $\Gamma$ ). Let $Y^{\circ} \subseteq Y$ be the complement of the union of the mirrors of $\Gamma$. Daniel Allcock has conjectured that the orbifold fundamental group of $Y^{\circ} / \Gamma$ surjects onto the bimonster.

In this article we study the reflection group $\Gamma$. Our main result shows that there is homomorphism from the Artin group of $D$ to the orbifold fundamental group of $Y^{\circ} / \Gamma$, obtained by sending the Artin generators to the generators of monodromy around the mirrors of the generating reflections in $\Gamma$. This answers a question in Allcock's article "A monstrous proposal" and takes a step towards the proof of Allcock's conjecture. The finite group $\operatorname{PGL}\left(3, \mathbb{F}_{3}\right) \subseteq \operatorname{Aut}(D)$ acts on $Y$ and fixes a complex hyperbolic line pointwise. We show that the restriction of $\Gamma$-invariant meromorphic automorphic forms on $Y$ to the complex hyperbolic line fixed by $\mathrm{PGL}\left(3, \mathbb{F}_{3}\right)$ gives meromorphic modular forms of level 13.

\section{INTRODUCTION}

This article is a continuation of [5]. Here we continue our study of the reflection group of the complex Lorentzian Leech lattice. Before describing our results (see section 1.4), we briefly recall the context, which makes the study of this particular reflection group interesting.

1.1. Some notation and background. Let $\mathbb{D}$ be a graph with vertex set $\left\{x_{1}, \cdots\right.$, $x_{k}$. Let $\mathcal{A}(\mathbb{D})$ be the group generated by $k$ generators, also denoted by $x_{1}, \cdots, x_{k}$, and the relations

$$
\begin{aligned}
x_{i} x_{j} & =x_{j} x_{i} \text { if }\left\{x_{i}, x_{j}\right\} \text { is not an edge of } \mathbb{D}, \\
x_{i} x_{j} x_{i} & =x_{j} x_{i} x_{j} \text { if }\left\{x_{i}, x_{j}\right\} \text { is an edge of } \mathbb{D},
\end{aligned}
$$

for all $i$ and $j$. The group $\mathcal{A}(\mathbb{D})$ is called the Artin group of the graph $\mathbb{D}$. Let $\operatorname{Cox}(\mathbb{D}, n)$ be the quotient of $\mathcal{A}(\mathbb{D})$, obtained by imposing the relations $x_{i}^{n}=1$ for all $x_{i} \in \mathbb{D}$. In this notation, the Coxeter group of the graph $\mathbb{D}$ is $\operatorname{Cox}(\mathbb{D}, 2)$.

The wreath product of the monster simple group with $\mathbb{Z} / 2 \mathbb{Z}$ is known as the bimonster. Conway and Norton conjectured a simple presentation of the bimonster that later became the Ivanov-Norton theorem.

Received by the editors December 9, 2013 and, in revised form, April 15, 2014.

2010 Mathematics Subject Classification. Primary 11H56, 20F05, 20F55; Secondary 20D08, 20F36, 51M10.

Key words and phrases. Complex hyperbolic reflection group, Leech lattice, bimonster, Artin group, orbifold fundamental group, generator and relation. 
Theorem ([17], [18, [21]). Let $M_{666}$ be a graph, shaped like a "Y", with 16 vertices (6 in each hand including the central vertex). Label the successive vertices in the $i$-th hand by $a, b_{i}, c_{i}, d_{i}, e_{i}, f_{i}$, with a being the central vertex. Then $\operatorname{Cox}\left(M_{666}, 2\right)$ maps onto the bimonster, and the kernel is generated by the single relation $\left(a b_{1} c_{1} a b_{2} c_{2} a b_{3} c_{3}\right)^{10}=1$.

Using the Ivanov-Norton theorem, Conway et al. obtained a second presentation of the bimonster. Let $D$ be the incidence graph of the projective plane over the finite field $\mathbb{F}_{3}$. The graph $M_{666}$ is a maximal sub-tree of $D$, so there is a natural map from $\operatorname{Cox}\left(M_{666}, 2\right)$ to $\operatorname{Cox}(D, 2)$.

Theorem ([1]; also see [9], [10]). The surjection from $\operatorname{Cox}\left(M_{666}, 2\right)$ to the bimonster extends to a surjection from $\operatorname{Cox}(D, 2)$ to the bimonster. The kernel is generated by some explicitly described simple relations called "deflating the 12-gons".

We need a bit of notation to introduce the reflection group. Let $\omega=e^{2 \pi i / 3}$ and $\mathcal{E}=\mathbb{Z}[\omega]$. Define the $\mathcal{E}$-lattice $L$ to be the direct sum of the complex Leech lattice and a hyperbolic cell (see 2.4-2.5 of [5]). Equivalently, we may define $L$ to be the unique Hermitian lattice of signature $(13,1)$ defined over $\mathcal{E}$ such that $(2+\omega) L^{\prime}=L$ (where $L^{\prime}$ denotes the dual lattice of $\left.L\right)$. Let $Y=\mathbb{P}_{+}\left(L^{\mathbb{C}}\right)$ be the set of complex lines of positive norm in the complex vector space $L^{\mathbb{C}}=L \otimes_{\mathcal{E}} \mathbb{C}$. The space $Y$ is isomorphic to 13 dimensional complex hyperbolic space. The projective automorphism group $\mathbb{P} \operatorname{Aut}(L)$ acts faithfully on $Y$. We write $\Gamma=\mathbb{P} \operatorname{Aut}(L)$.

Daniel Allcock showed in [1 that the reflection group of $L$, denoted by $R(L)$, has finite index in $\operatorname{Aut}(L)$. So $R(L)$ is an arithmetic subgroup of $U(1,13)$. This example provides the largest dimension in which an arithmetic hyperbolic reflection group is known. Allcock also observed that there is a map $\phi: \operatorname{Cox}\left(M_{666}, 3\right) \rightarrow R(L)$ sending the generators to complex reflections of order 3 . While trying to better understand the reflection group of $L$, we proved the following theorem:

Theorem $1.2([5])$. (a) The map $\phi: \operatorname{Cox}\left(M_{666}, 3\right) \rightarrow R(L)$ is onto and it extends to a surjection $\phi: \operatorname{Cox}(D, 3) \rightarrow R(L)$. In other words, there are 26 complex reflections of order 3 (called simple reflections) in the reflection group of $L$ which braid or commute according to the diagram $D$, and these reflections generate $R(L)$. (The proof of this fact, given in [5], used a computer calculation. Since then, Allcock has found a computer-free proof; see [3.)

(b) One has $\operatorname{Aut}(L)=R(L)$. The "deflation relation" holds in $R(L)$.

(c) (See Prop. 6.1 in [5]) Let $D^{\perp}$ be the set of 26 mirrors, fixed by the 26 simple reflections. The group $\Gamma$ possesses a subgroup $Q \simeq \operatorname{PGL}\left(3, \mathbb{F}_{3}\right) .2$ (called the group of diagram automorphisms) which permutes the mirrors $D^{\perp}$ in the same way as $\operatorname{PGL}\left(3, \mathbb{F}_{3}\right) .2$ permutes the points and lines of $\mathbb{P}^{2}\left(\mathbb{F}_{3}\right)$. The action of $Q$ fixes a unique point $\bar{\rho}$ in the complex hyperbolic space $Y$. The set $D^{\perp}$ consists of precisely the mirrors that are closest to $\bar{\rho}$.

We are interested in understanding the relationship between $R(L)$ and the bimonster. Daniel Allcock has made a conjecture to explain this relationship using complex hyperbolic geometry. (In fact the conjecture predates [5].)

Conjecture 1.3 (Allcock; see [2], [5]). Consider the action of $\Gamma=\mathbb{P} \operatorname{Aut}(L)$ on the complex hyperbolic space $Y$. Let $\mathcal{M}$ be the union of the fixed points of the reflections in $\Gamma$. Let $Y^{\circ}=Y \backslash \mathcal{M}$. Then the orbifold fundamental group of $Y^{\circ} / \Gamma$ maps onto the bimonster. 
See 2] for more on this conjecture and its possible ramifications. There are speculative ideas explored in [2] regarding a possible candidate for the conjectured monster manifold and an interpretation of $Y / \Gamma$ as a nice moduli space.

1.4. Summary of results. In section 2, we recall some basic definitions and set up our notation. We explain how to construct our lattice $L$ from incidence geometry of $\mathbb{P}^{2}\left(\mathbb{F}_{3}\right)$.

Section 3 recalls the results from [5] that we need, in some detail. Lemma 3.2 and Theorem 5.8 of [5] show that $\operatorname{Aut}(L)$ can be generated by 16 complex reflections of order 3, making an $M_{666}$ diagram. In section 3 , we improve this by showing that 14 complex reflections of order 3 suffice to generate the reflection group of $L$. Since $L$ is 14 dimensional, these 14 reflections, making a $M_{655}$ diagram, form a minimal set of generators for $\operatorname{Aut}(L)$.

Section 4 contains the bulk of our work. Here we study the fundamental group of the orbifold $Y^{\circ} / \Gamma$, which we denote by $G$. By the definition of $G$ (see Definition 4.3), there is an exact sequence

$$
1 \rightarrow \pi_{1}\left(Y^{\circ}\right) \rightarrow G \stackrel{\pi_{\Gamma}^{G}}{\rightarrow} \Gamma \rightarrow 1
$$

By the results from [5] quoted above, we have a surjection $\phi$ from $\mathcal{A}(D)$ to $\Gamma$. The main result of section 4 is Theorem 4.4, which says that the map $\phi$ lifts to a homomorphism $\psi: \mathcal{A}(D) \rightarrow G$. This answers a question asked by Allcock in [2].

To put this theorem in context, we should remark that we expect the fundamental group $G$ to be something like the Artin group $\mathcal{A}(D)$, maybe with some extra relations. This expectation is based on the analogy of our reflection group $R(L)$ with the Weyl groups and the theorem of Brieskorn, Saito [8] and Deligne [13] on fundamental groups of complements of complex hyperplane arrangements associated to Weyl groups. The analogy between the reflection group $R(L)$ and Weyl groups has been a useful guiding principle in this project.

Next we would like to explain how Theorem 4.4 fits in a plan towards proving Conjecture 1.3 and explain the present status of this conjecture and its geometric implication. Let $\tilde{Y}^{\circ}$ be the universal cover of $Y^{\circ}$. The group $G$ acts as deck transformations on the ramified covering $\left(\tilde{Y}^{\circ} \rightarrow Y^{\circ} / \Gamma\right)$. Let $G_{1} \subseteq G$ be the image of the map $\psi$. Let $N$ be the normal subgroup of $G_{1}$ generated by $\left\{\psi(r)^{2}: r \in D\right\}$. Then we have the following tower of ramified coverings:

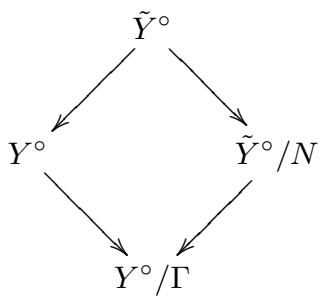

By Theorem 4.4, there is a surjection from $\operatorname{Cox}(D, 2)$ to $G_{1} / N$, and $G_{1} / N$ acts naturally as deck transformations on the (possibly ramified) covering $\left(\tilde{Y}^{\circ} / N \rightarrow\right.$ $\left.Y^{\circ} / \Gamma\right)$. The author has been working jointly with Allcock on a project that aims to show that $G_{1}=G 1$ A sketch of a geometric argument to prove that the deflation

\footnotetext{
${ }^{1}$ We have an essentially complete proof of this. We are being cautious since the proof is long and complicated and part of it is still not in print. Part of the proof uses refinements of methods we use to prove our Theorem 4.4 namely, constructing explicit 2-cells in $Y^{\circ}$ with given boundary
} 
relations hold in $G / N$ was explained to me by Daniel Allcock. Also recent work of Heckman and Reiken [15, 16] makes it plausible that the deflation relation holds in $G / N$. In view of these developments, if we can show that $G / N$ has at least 3 elements, then we would know that $G / N$ is the bimonster, thus proving Allcock's conjecture and producing a complex 13 dimensional orbifold with an action of the bimonster. At present, showing $|G / N|>2$ seems hard.

We prove Theorem 4.4 by constructing explicit homotopies between paths in $Y^{\circ}$ to obtain relations in the group $G$. Let $\gamma_{1}$ and $\gamma_{2}$ be two paths in $Y^{\circ}$ with the same beginning and endpoints. To construct a homotopy from $\gamma_{1}$ to $\gamma_{2}$ in $Y^{\circ}$, we construct a 2-cell $C^{\prime} \subseteq Y$ whose boundary is $\gamma_{1} \cup \gamma_{2}$ and then show that $C^{\prime}$ does not intersect the mirrors of reflection. The main tool to show that $C^{\prime}$ avoids the mirrors is to use Theorem 1.2(c), which provides some information about the configuration of mirrors near the point $\bar{\rho} \in Y^{\circ}$ fixed by the group $\operatorname{PGL}\left(3, \mathbb{F}_{3}\right) .2$ of diagram automorphisms. This information about the mirror arrangement, together with some complex hyperbolic geometry, lets us restrict the possible set of mirrors intersecting $C^{\prime}$ to a finite set. The proof is completed by directly checking that the remaining finite set of mirrors does not intersect $C^{\prime}$.

The unique point $\bar{\rho}$ fixed by the group $Q \simeq \operatorname{PGL}\left(3, \mathbb{F}_{3}\right) .2$ of diagram automorphisms plays an important role in all the major arguments in [5] as well as in the present article. In view of this, in section 5 we make a detailed study of the 2 dimensional lattice $F$ fixed by the group $\operatorname{PGL}\left(3, \mathbb{F}_{3}\right) \subseteq Q$; see Theorem 5.1 . At the time of writing this section, we expected that Theorem 5.1 would be useful in further study of the fundamental group of $\pi_{1}\left(Y^{\circ} / \Gamma\right)$. Indeed, we have since found a use for Theorem 5.1 while proving (ongoing joint work with Allcock) that the images of the 26 simple reflections under the map $\psi$ generate $\pi_{1}\left(Y^{\circ} / \Gamma\right)$.

The complex hyperbolic line $\mathbb{P}_{+}\left(F^{\mathbb{C}}\right)$ is isometric to the upper half plane $\mathcal{H}^{2}$. We construct an explicit isometry $\beta: \mathbb{P}_{+}\left(F^{\mathbb{C}}\right) \rightarrow \mathcal{H}^{2}$ such that "cusps of Leech type" map to 0 and $\infty$ and $\bar{\rho}$ maps to $\sqrt{-1}$. Let $\Gamma_{F}$ be the set of elements of $\Gamma$ that fix $F$ as a set. The isometry $\beta$ induces a group homomorphism $c_{\beta}: \Gamma_{F} \rightarrow \mathrm{PSL}_{2}(\mathbb{R})$. We show that the image of $c_{\beta}$ contains the principal congruence subgroup $\Gamma(13)$. The group $c_{\beta}\left(\Gamma_{F}\right) \cap \mathrm{PSL}_{2}(\mathbb{Z})$ has genus zero. In fact it is a conjugate of $\Gamma_{0}(13)$. The diagram automorphism $\sigma$ that corresponds to interchanging points and lines of $\mathbb{P}^{2}\left(\mathbb{F}_{3}\right)$ acts as the involution $\left(\tau \mapsto-\tau^{-1}\right)$.

This result provides an explicit way to obtain ordinary modular forms from automorphic forms of type $U(1,13)$ defined on the Hermitian symmetric space $Y$. Let $f$ be a meromorphic automorphic form on $\mathbb{C} H^{13}$ automorphic with respect to the group $\Gamma$, with zeroes and poles along the mirrors of reflection. From Theorem 1.2(c), we know that the complex hyperbolic line $\mathbb{P}_{+}\left(F^{\mathbb{C}}\right)$ is not contained in any mirror, so the restriction of $f$ to $\mathbb{P}_{+}\left(F^{\mathbb{C}}\right)$ is well-defined and non-zero; thus it is a meromorphic modular form of level 13. Meromorphic automorphic forms on $Y$ with zeros and poles along the mirrors of reflection can be constructed by the Borcherds method of singular theta lift (see [7] for the general method and [1] for our example). Alternatively, as we shall see in Remark 5.3. one can define such automorphic forms as explicit infinite series similar to the Eisenstein series (also see [20], where these are called the Poincaré-Weierstrass series). These automorphic forms may be useful in obtaining an explicit projective uniformization of $Y^{\circ} / \Gamma$.

curves and checking how the mirrors intersect these 2-cells to construct homotopies between paths. The first part of the joint work is in press 4]; the rest is being written up. 
1.5. Index of some commonly used notation. We use the Atlas notation for groups.

$\mathcal{A}(D)$ the Artin group of the diagram $D$.

$\operatorname{Aut}(L)$ automorphism group of the lattice $L$.

$\beta \quad$ an isometry from $\mathbb{P}_{+}\left(F^{\mathbb{C}}\right)$ to $\mathcal{H}^{2}$ or the matrix $\left(\begin{array}{cc}p & \omega \\ 1 & \bar{p}\end{array}\right)$ that represents it.

$\mathbb{C} H^{n}$ the complex hyperbolic space of dimension $n$.

$D \quad$ the incidence graph of $P^{2}\left(\mathbb{F}_{3}\right)$ or the set of 26 simple roots of $L$ labeled by vertices of this graph.

$D(K)$ the discriminant group of a lattice $K$; i.e., $D(K)=K^{\prime} / K$.

$\mathcal{E} \quad=\mathbb{Z}\left[e^{2 \pi i / 3}\right]$.

$\epsilon \quad$ a small positive real number.

$F \quad$ the sub-lattice of $L$ fixed by the group $\operatorname{PGL}\left(3, \mathbb{F}_{3}\right)$ of diagram automorphisms.

$G \quad$ the orbifold fundamental group of $X^{\circ}$.

$\Gamma \quad$ the automorphism group of $L$ modulo scalars: $\Gamma=\mathbb{P} \operatorname{Aut}(L)$.

ht $(r) \quad$ height of a vector $r$, given by $\operatorname{ht}(r)=|\langle\bar{\rho}, r\rangle| /|\bar{\rho}|^{2}$.

$l \quad$ an element of $\mathcal{L}$.

$L \quad$ the complex Leech lattice plus a hyperbolic cell, defined over $\mathcal{E}$.

$L^{\prime} \quad$ the dual lattice of $L$.

$\mathcal{L} \quad$ the set of lines of $\mathbb{P}^{2}\left(\mathbb{F}_{3}\right)$ or the simple roots of $L$ that correspond to them.

$\mathcal{M}$ the union of the mirrors of the reflection group of $L$.

$p \quad=2+\omega$.

$p_{1} \quad=3-\omega$.

$\mathcal{P} \quad$ the set of points of $\mathbb{P}^{2}\left(\mathbb{F}_{3}\right)$ or the simple roots of $L$ that correspond to them.

$\phi_{r} \quad \omega$-reflection in the vector $r$.

$Q \quad$ the group of diagram automorphisms acting on $Y$; one has $Q \simeq$ $\operatorname{PGL}\left(3, \mathbb{F}_{3}\right) .2$.

$r_{i} \quad r_{1}, \cdots, r_{26}$ are the simple roots.

$\rho_{i} \quad \rho_{i}=r_{i}$ if $r_{i} \in \mathcal{P}$, and $\rho_{i}=\xi r_{i}$ if $r_{i} \in \mathcal{L}$.

$R(L) \quad$ (complex) reflection group of the lattice $L$.

$[\bar{\rho}] \quad$ the unique point in the complex hyperbolic space $Y$, fixed by $Q$ (see (6) ).

$\sigma \quad$ a diagram automorphism that corresponds to interchanging the points and lines of $\mathbb{P}^{2}\left(\mathbb{F}_{3}\right)$.

$\theta \quad=\omega-\bar{\omega}$.

$\omega \quad=e^{2 \pi i / 3}$.

$\left[w_{\mathcal{P}}\right]$ the point of $Y$ where the 13 mirrors $\left\{x^{\perp}: x \in \mathcal{P}\right\}$ meet (see equation (44)).

$\left[w_{\mathcal{L}}\right] \quad$ the point of $Y$ where the 13 mirrors $\left\{l^{\perp}: l \in \mathcal{L}\right\}$ meet (see equation (44)).

$x \quad$ an element of $\mathcal{P}$.

$\xi \quad=e^{-\pi i / 6}$.

$X^{\circ} \quad$ the orbifold $Y^{\circ} / \Gamma$.

$Y \quad$ the set of complex lines of positive norm in $L \otimes_{\mathcal{E}} \mathbb{C}$. $\left(\right.$ Note: $Y \simeq \mathbb{C} H^{13}$.)

$Y^{\circ} \quad=Y \backslash \mathcal{M}$.

\section{Some Generalities on COMPlex HYPERBolic REFleCtion Groups}

2.1. The complex hyperbolic space. A general reference for complex hyperbolic geometry is [14]. Let $V$ be a complex vector space, with a Hermitian form $\langle$,$\rangle .$ 
Assume that $V$ is Lorentzian; that is, the Hermitian form on $V$ has signature $(1, m-1)$. Then the open subset $\mathbb{P}_{+}(V)$ of the projective space $\mathbb{P}(V)$, consisting of the complex lines of positive norm, is called the complex hyperbolic space of $V$. If $H \subseteq V$, then let $[H]$ be the subset of $\mathbb{P}_{+}(V)$ or $\mathbb{P}(V)$ determined by $H$. We shall often abuse notation and write $H$ for $[H]$ if there is no possibility of confusion.

Let $\mathbb{C}^{m, n}$ denote the complex vector space $\mathbb{C}^{m+n}$ with the Hermitian form

$$
\langle z, w\rangle=\sum_{i=1}^{m} \bar{z}_{i} w_{i}-\sum_{i=m+1}^{m+n} \bar{z}_{i} w_{i} .
$$

The $n$ dimensional complex hyperbolic space $\mathbb{C} H^{n}=\mathbb{P}_{+}\left(\mathbb{C}^{1, n}\right)$ is homeomorphic to the unit ball $B^{n}(\mathbb{C}) \subseteq \mathbb{C}^{n}$ via the isomorphism $b^{\prime}:\left[z_{0}, \cdots, z_{n}\right]^{\prime} \mapsto\left(z_{1} / z_{0}, \cdots, z_{n} / z_{0}\right)$. Let $x$ and $y$ be two vectors in $V$ having positive norm. The metric on $\mathbb{C} H^{n}$ is given by

$$
d([x],[y])=\cosh ^{-1}\left(\frac{|\langle x, y\rangle|}{|x| \cdot|y|}\right) .
$$

This differs from the metric used in [14] by a factor of 2 , but the scaling of the metric is not important for us.

2.2. The complex hyperbolic line and the real hyperbolic plane. We need to consider two models of the real hyperbolic plane, namely the unit ball $B^{1}(\mathbb{C})$ and the upper half plane $\mathcal{H}^{2}$. It will be convenient to identify both as subsets of $\mathbb{P}^{1}(\mathbb{C})$ by $\tau \mapsto\left(\begin{array}{c}\tau \\ 1\end{array}\right)$.

The map $b^{\prime}:\left[z_{0}, z_{1}\right] \rightarrow z_{1} / z_{0}$ defines an isometry between the complex hyperbolic line $\mathbb{C} H^{1}$ and the (ball model of) real hyperbolic plane. Let $\mathcal{H}^{2}$ be the upper half plane with the Poincaré metric: $d\left(\tau, \tau^{\prime}\right)=\cosh ^{-1}\left(2^{-1} \operatorname{Im}(\tau)^{-\frac{1}{2}} \operatorname{Im}\left(\tau^{\prime}\right)^{-\frac{1}{2}}\left|\tau^{\prime}-\bar{\tau}\right|\right)$. Let $C: B^{1}(\mathbb{C}) \rightarrow \mathcal{H}^{2}$ denote the Cayley isomorphism: $C(w)=i\left(\frac{1+w}{1-w}\right)$. The composition

$$
b=C \circ b^{\prime}: \mathbb{P}_{+}\left(\mathbb{C}^{1,1}\right) \rightarrow \mathcal{H}^{2}
$$

is an isometry. Let $S=\left(\begin{array}{cc}0 & -1 \\ 1 & 0\end{array}\right)$ and $T=\left(\begin{array}{ll}1 & 1 \\ 0 & 1\end{array}\right)$ be the standard generators of $\mathrm{SL}_{2}(\mathbb{Z})$.

2.3. Complex lattices and their reflection groups. We recall our notation regarding complex reflection groups. In most places, we maintain the notation of [5] and refer the reader to section 2.2 of [5] for more details. Let $\xi=e^{-\pi i / 6}$, $\omega=-\xi^{2}$ and $\mathcal{E}=\mathbb{Z}[\omega]$. An $\mathcal{E}$-lattice $K$ is a free $\mathcal{E}$-module of finite rank, together with an $\mathcal{E}$-valued Hermitian form $\langle\rangle:, K \times K \rightarrow \mathcal{E}$ (always linear in the second variable). Let $K^{\mathbb{C}}=K \otimes_{\mathcal{E}} \mathbb{C}$ be the underlying Hermitian vector space of $K$. We shall mainly be concerned with a lattice $L$ for which $L^{\mathbb{C}} \simeq \mathbb{C}^{1,13}$. So the definite lattices that we consider will always be negative definite. Let $K^{\prime}$ be the dual lattice of $K$, defined by $K^{\prime}=\left\{v \in K^{\mathbb{C}}:\langle v, x\rangle \in \mathcal{E}\right.$ for all $\left.x \in K\right\}$. Let $\mathrm{D}(K)=K^{\prime} / K$ be the discriminant group of $K$.

Let $r$ be a primitive vector of $K$ having negative norm, that is, $|r|^{2}:=\langle r, r\rangle<0$. Let $\alpha$ be a root of unity in $\mathcal{E}, \alpha \neq 1$. The complex reflection $\phi_{r}^{\alpha} \in \operatorname{Aut}(K)$ is the automorphism of $K$ that fixes the hyperplane $r^{\perp}$ orthogonal to $r$ and multiplies $r$ by $\alpha$. The vector $r$ is called the root of the reflection, and the hyperplane $r^{\perp}$ (or its image in the projective space $\mathbb{P}\left(K^{\mathbb{C}}\right)$ ) is called the mirror of the reflection. The reflection group of $K$, denoted by $R(K)$, is the subgroup of $\operatorname{Aut}(K)$ generated by reflections in the roots of $K$. We write $\phi_{r}=\phi_{r}^{\omega}$ and call it the $\omega$-reflection in $r$. 
2.4. The incidence graph of $\mathbb{P}^{2}\left(\mathbb{F}_{3}\right)$. The projective plane over $\mathbb{F}_{3}$ has 13 points and 13 lines. Let $\mathcal{P}$ be the set of points and $\mathcal{L}$ be the set of lines. If a point $x \in \mathcal{P}$ is incident on a line $l \in \mathcal{L}$, then we write $x \in l$. Let $D$ be the (directed) incidence graph of $\mathbb{P}^{2}\left(\mathbb{F}_{3}\right)$. The vertex set of $D$ is $\mathcal{P} \cup \mathcal{L}$. There is a directed edge in $D$ from a vertex $l$ to a vertex $x$ if $x \in \mathcal{P}, l \in \mathcal{L}$ and $x \in l$.

2.5. Definition of the Lorentzian lattice from geometry of $\mathbb{P}^{2}\left(\mathbb{F}_{3}\right)$. Let $p=$ $2+\omega$. Let $L^{\circ}$ be the free $\mathcal{E}$-module of rank 26 with basis vectors indexed by $D=\mathcal{P} \cup \mathcal{L}$. Let $x, x^{\prime} \in \mathcal{P}$ and $l, l^{\prime} \in \mathcal{L}$. Define a Hermitian for an $L^{\circ}$ by

$$
\left\langle x, x^{\prime}\right\rangle=\left\{\begin{array}{lc}
-3 & \text { if } x=x^{\prime}, \\
0 & \text { otherwise }
\end{array} \quad\left\langle l, l^{\prime}\right\rangle=\left\{\begin{array}{ll}
-3 & \text { if } l=l^{\prime}, \\
0 & \text { otherwise }
\end{array} \quad\langle x, l\rangle= \begin{cases}p & \text { if } x \in l \\
0 & \text { otherwise }\end{cases}\right.\right.
$$

The lattice $L^{\circ}$ has a 12 dimensional radical. To see this, let $w_{l}=\bar{p} l+\sum_{x \in l} x$ for any $l \in \mathcal{L}$. Then using (2) and the geometry of $\mathbb{P}^{2}\left(\mathbb{F}_{3}\right)$ one easily checks that

$$
\left\langle x^{\prime}, w_{l}\right\rangle=0 \text { and }\left\langle w_{l}, l^{\prime}\right\rangle=p \quad \text { or all } \quad x^{\prime} \in \mathcal{P} \text { and } l^{\prime} \in \mathcal{L} .
$$

So if $l_{1}, l_{2} \in \mathcal{L}$, then $\left(w_{l_{1}}-w_{l_{2}}\right)$ is in the radical $\operatorname{Rad}\left(L^{\circ}\right)$. So $\operatorname{rank}\left(\operatorname{Rad}\left(L^{\circ}\right)\right) \geq 12$. Define

$$
L_{3}=L^{\circ} / \operatorname{Rad}\left(L^{\circ}\right) .
$$

The Hermitian form on $L^{\circ}$ descends to a Hermitian form on $L_{3}$, which is again denoted by $\langle$,$\rangle . Let w_{\mathcal{P}}$ be the image of $w_{l}$ in $L_{3}$ (for any $l \in \mathcal{L}$ ). Equation (3) implies that the vector $w_{\mathcal{P}}$ is orthogonal (in $L_{3}$ ) to each $x \in \mathcal{P}$ and $\left\langle w_{\mathcal{P}}, l\right\rangle=p$ for all $l \in \mathcal{L}$. It follows that the matrix of dot products of the vectors $\mathcal{P} \cup\left\{w_{\mathcal{P}}\right\}$ is a diagonal matrix with diagonal entries $(-3,-3, \cdots,-3,3)$. So $\operatorname{rank}(L) \geq 14$. It follows that $\operatorname{rank}\left(\operatorname{Rad}\left(L^{\circ}\right)\right)=12, \operatorname{rank}\left(L_{3}\right)=14$ and $L_{3}$ is a non-degenerate $\mathcal{E}$-module of signature $(1,13)$.

Remark 2.1. From the properties of the incidence matrix of $\mathbb{P}^{2}\left(\mathbb{F}_{3}\right)$ one can prove that $L_{3}$ is $p$-modular, that is, $p L_{3}^{\prime}=L_{3}$. The definition of $L_{3}$ given in section 2.5 can be mimicked for finite projective planes $\mathbb{P}^{2}\left(\mathbb{F}_{q}\right)$ for other values of $q$ and number rings other than $\mathcal{E}$. This way one obtains infinitely many $\sqrt{-q}$-modular Lorentzian Hermitian lattices; see $\left[6\right.$. If $q \equiv 3 \bmod 4$ is a rational prime and $\left(q^{2}+q+1\right)$ is a norm of some element in $\mathbb{Q}[\sqrt{-q}]$, then we get an even unimodular $2 q(q+1)$ dimensional $\mathbb{Z}$-lattice whose symmetry group contains $\operatorname{PGL}\left(3, \mathbb{F}_{q}\right)$. The first one among these lattices is the Leech lattice.

\section{The Reflection Group of the LoRentzian LeECh LATtice}

3.1. Chiefly to set up notation, we need to recall a few results from [5] in some detail. Then we prove a couple of small lemmas. As before, let $L$ be the direct sum of the complex Leech lattice and a hyperbolic cell. Let $L_{3}$ be the lattice defined in section 2.5

Observation. Since both $L$ and $L_{3}$ have signature $(1,13)$ and satisfy $p L^{\prime}=L$ and $p L_{3}^{\prime}=L_{3}$, it follows that $L \simeq L_{3}$. This can also be directly proved by showing that the lattice $L$ contains 26 vectors of norm -3 (called the simple roots) that have inner products prescribed by (2) (see 3.1 of [5]). 
We want to study the action of $\Gamma=\mathbb{P} \operatorname{Aut}(L)$ on $Y=\mathbb{P}_{+}\left(L^{\mathbb{C}}\right) \simeq \mathbb{C} H^{13}$. Note that the simple roots of $L$ correspond to the vertices of the graph $D$. A vertex of $D$ and the corresponding simple root of $L$ are denoted by the same symbol. If $\{r, s\}$ is an edge of $D$, then the $\omega$-reflections in the simple roots $r$ and $s$ braid. If $\{r, s\}$ is not an edge, then the reflections commute. As mentioned in Theorem 1.2(a), the order 3 complex reflections in the 26 simple roots generate $\operatorname{Aut}(L)$.

The construction of $L$ given in section 2.5 implies that the group of diagram automorphisms $Q \simeq \operatorname{PGL}\left(3, \mathbb{F}_{3}\right) .2$ acts on the complex hyperbolic space $Y=\mathbb{P}_{+}\left(L^{\mathbb{C}}\right)$. The action of $\operatorname{PGL}\left(3, \mathbb{F}_{3}\right)$ on $L$ pointwise fixes a 2 dimensional primitive sub-lattice $F$ of $L$ having a basis $w=\left(w_{\mathcal{P}}, w_{\mathcal{L}}\right)$ where

$$
w_{\mathcal{P}}=\bar{p} l+\sum_{x^{\prime} \in l} x^{\prime} \quad \text { and } \quad w_{\mathcal{L}}=p x+\sum_{x \in l^{\prime}} l^{\prime}
$$

for any $x \in \mathcal{P}$ and $l \in \mathcal{L}$. We shall make a detailed study of the lattice $F$ in section 5. From the inner products given in (2), it is easy to check that for all $x \in \mathcal{P}$ and $l \in \mathcal{L}$, we have

$$
\left\langle w_{\mathcal{P}}, x\right\rangle=\left\langle w_{\mathcal{L}}, l\right\rangle=0,\left\langle w_{\mathcal{P}}, l\right\rangle=\left\langle x, w_{\mathcal{L}}\right\rangle=p \text {, and }\left(\begin{array}{c}
\left\langle w_{\mathcal{P}}, w_{\mathcal{P}}\right\rangle\left\langle w_{\mathcal{P}}, w_{\mathcal{L}}\right\rangle \\
\left\langle w_{\mathcal{L}}, w_{\mathcal{P}}\right\rangle\left\langle w_{\mathcal{L}}, w_{\mathcal{L}}\right\rangle
\end{array}\right)=\left(\begin{array}{cc}
3 & 4 p \\
4 \bar{p} & 3
\end{array}\right) .
$$

Let $D^{\perp}$ be the set of mirrors perpendicular to the simple roots. These are called the simple mirrors. Equation (5) implies that the 13 simple mirrors in $\mathbb{C} H^{13}$ corresponding to the elements of $\mathcal{P}($ resp. $\mathcal{L})$ meet at $\left[w_{\mathcal{P}}\right]\left(\right.$ resp. $\left.\left[w_{\mathcal{L}}\right]\right)$.

Let $\left(r_{1}, \cdots, r_{26}\right)$ be the 26 roots that form the vertices of $D$. Let $\rho_{1}, \cdots, \rho_{26}$ be defined by $\rho_{j}=r_{j}$ if $r_{j} \in \mathcal{P}$ and $\rho_{j}=\xi r_{j}$ if $r_{j} \in \mathcal{L}$. There exists $\sigma \in \operatorname{Aut}(L)$ (cf. [5], Section 5.3 2 that corresponds to interchanging points and lines of $\mathbb{P}^{2}\left(\mathbb{F}_{3}\right)$. The "point-line involution" $\sigma$ takes $\rho_{j}$ to $\xi \rho_{\pi(j)}$ where $\pi$ is some order 2 permutation of the 26 indices such that if $r_{j} \in \mathcal{P}$, then $r_{\pi(j)} \in \mathcal{L}$ and vice versa. Let $Q$ be the group of automorphisms of $D$ as a graph. We call $Q$ the group of diagram automorphisms. One has $Q \simeq \operatorname{PGL}\left(3, \mathbb{F}_{3}\right) .2$. The group $Q$ contains $\operatorname{PGL}\left(3, \mathbb{F}_{3}\right)$ as an index 2 subgroup, and $\sigma$ generates the quotient $\mathbb{Z} / 2 \mathbb{Z}$. Let

$$
\bar{\rho}=\frac{1}{26}\left(\sum_{x \in \mathcal{P}} x+\xi \sum_{l \in \mathcal{L}} l\right)=\frac{1}{26} \sum_{j=1}^{26} \rho_{j}=\frac{w_{\mathcal{P}}+\xi w_{\mathcal{L}}}{2(4+\sqrt{3})} .
$$

Then $[\bar{\rho}]$ is the midpoint of the geodesic joining $\left[w_{\mathcal{P}}\right]$ and $\left[w_{\mathcal{L}}\right]$. The diagram automorphism $\sigma$ interchanges $\left[w_{\mathcal{P}}\right]$ and $\left[w_{\mathcal{L}}\right]$, so $[\bar{\rho}]$ is the only point in $\mathbb{C} H^{13}$ fixed by $Q$. Since $Q$ fixes $[\bar{\rho}]$ and acts transitively on the simple mirrors, it follows that $[\bar{\rho}]$ is equidistant from the simple mirrors. Let $d_{0}=d\left(r^{\perp},[\bar{\rho}]\right)$ for $r \in D$. As mentioned in Theorem $1.2(\mathrm{c})$, we know that $d\left(r^{\perp},[\bar{\rho}]\right) \geq d_{0}$ for all roots $r$ of $L$ and $d\left(r^{\perp},[\bar{\rho}]\right)=d_{0}$ if and only if $r^{\perp}$ is a simple mirror. In other words, the simple mirrors are closest to $[\bar{\rho}]$ and all other mirrors are further away.

Lemma 3.2. Consider $\Gamma=\mathbb{P} \operatorname{Aut}(L)$ acting on $Y \simeq \mathbb{C} H^{13}$. Then the stabilizer (in $\Gamma$ ) of the set $D^{\perp}$ and the stabilizer of $[\bar{\rho}]$ are both equal to $Q \simeq \operatorname{PGL}\left(3, \mathbb{F}_{3}\right) .2$.

Proof. If $g \in \mathbb{P} \operatorname{Aut}(L)$ fixes $D^{\perp}$ as a set, then it must fix $[\bar{\rho}]$, it being the only point equidistant from each simple mirror. Conversely, if $g \in G$ fixes $[\bar{\rho}]$, then it

\footnotetext{
${ }^{2}$ Caution: Our notation here differs slightly from [5]. In [5], $r_{1}, \cdots, r_{26}$ were specific roots such that $r_{1}, \cdots, r_{13}$ were the points and $r_{14}, \cdots, r_{26}$ were the lines. Here we do not make this assumption, because later we shall work with an arbitrary pair of roots in $D$ which we shall call $r_{1}$ and $r_{2}$.
} 
must permute the mirrors in $D^{\perp}$ in some way and thus determine an element of $\operatorname{PGL}\left(3, \mathbb{F}_{3}\right) .2$. So the stabilizer of the set $D^{\perp}$ and the point $[\bar{\rho}]$ are equal.

Suppose $g$ acts trivially on $D^{\perp}$. Let $\tilde{g}$ be a lift of $g$ in $\operatorname{Aut}(L)$. For each $r \in D$, it follows that $\tilde{g} r=\mu_{r} r$ for some root of unity $\mu_{r}$. If $r$ and $s$ are two simple roots with $\langle r, s\rangle=p$, then $p=\langle\tilde{g} r, \tilde{g} s\rangle=\bar{\mu}_{r} \mu_{s}\langle r, s\rangle=\bar{\mu}_{r} \mu_{s} p$. So $\mu_{r}=\mu_{s}$ whenever there is an edge between $r$ and $s$ in the graph $D$, which implies $\mu_{r}=\mu$ is a constant. So $g$ is equal to the identity.

In [5], we showed that $\operatorname{Aut}(L)$ is generated by the 16 simple reflections in the roots $\left\{a, b_{j}, c_{j}, d_{j}, e_{j}, f_{j}: j=1,2,3\right\}$ that form the $M_{666}$ diagram where $a$ is the central vertex of the " $Y$ "-shaped diagram $M_{666}$ and $a, b_{j}, c_{j}, d_{j}, e_{j}, f_{j}$ are the successive vertices of the three hands of the $Y$-shaped diagram. We shall end this section by noting a small improvement of this fact.

Lemma 3.3. The automorphism group of $L$ is generated by the $14 \omega$-reflections in the simple roots $a, f_{1}$ and $b_{i}, c_{i}, d_{i}, e_{i}$ for $i=1,2,3$. Since $L$ is 14 dimensional, the 14 simple reflections of a $M_{655}$ diagram form a minimal set of generators for $\operatorname{Aut}(L)$.

The proof of the lemma uses the deflation relations, which we now recall. By a sub-graph of $D$ we mean the graph formed by taking a subset of vertices of $D$ and all the edges between these vertices in $D$. A 12-gon in $D$ is a sub-graph of $D$ that has the shape of a circuit of length 12 (in other words, an affine $A_{11}$ diagram). Let $y$ be a 12 -gon in $D$ and let $\left\{y_{1}, \cdots, y_{12}\right\}$ be the $\omega$-reflections successive vertices of $y$. Consider the relation

$$
\left(y_{1} y_{2} \cdots y_{10}\right) y_{11}\left(y_{1} y_{2} \cdots y_{10}\right)^{-1}=y_{12} \text {. }
$$

Following [11, we call this relation "deflate $(y)$ ". The affine Coxeter group of type $A_{11}$ generated by $y_{1}, \cdots, y_{12}$ reduces to the symmetric group $S_{12}$ in the presence of the relation deflate $(y)$. The bimonster is the quotient of $\operatorname{Cox}(D, 2)$ obtained by adding the relations deflate $(y)$ for all free 12-gon $y$ in $D$ (see [11).

Proof of Lemma 3.3. There exists a 12-gon $y$ in $D$ such that (7) holds. For example, in the notation of [11, one may choose the roots $\left(f_{2}, e_{2}, d_{2}, c_{2}, b_{2}, a, b_{1}, c_{1}, d_{1}, e_{1}\right.$, $\left.f_{1}, a_{3}\right)$ to be the successive vertices of $y$ and one verifies that

$$
\phi_{f_{2}} \phi_{e_{2}} \phi_{d_{2}} \phi_{c_{2}} \phi_{b_{2}} \phi_{a} \phi_{b_{1}} \phi_{c_{1}} \phi_{d_{1}} \phi_{e_{1}}\left(f_{1}\right)=\omega^{2} a_{3},
$$

which implies that equation (17) holds for the 12-gon $y$. It is an amusing exercise to show that the group $\operatorname{PGL}\left(3, \mathbb{F}_{3}\right) .2$ acts transitively on the set of marked 12-gons in $D$. So the relation (7) holds for any 12 -gon in $D$. The deflation relation implies that

$$
y_{12}=\left(y_{1} \cdots y_{10}\right) y_{11}\left(y_{1} \cdots y_{10}\right)^{-1}=\left(y_{11} \cdots y_{2}\right) y_{1}\left(y_{11} \cdots y_{2}\right)^{-1} .
$$

Moving the $y_{1}$ 's to the other side of the equation and using the commuting relations, we get

$$
\begin{aligned}
\left(y_{2} \cdots y_{10}\right) y_{11}\left(y_{2} \cdots y_{10}\right)^{-1} & =y_{1}^{-1}\left(y_{11} \cdots y_{2}\right) y_{1}\left(y_{11} \cdots y_{2}\right)^{-1} y_{1} \\
& =\left(y_{11} \cdots y_{3}\right) y_{1}^{-1} y_{2} y_{1} y_{2}^{-1} y_{1}\left(y_{11} \cdots y_{3}\right)^{-1} .
\end{aligned}
$$

Using the braiding relations $y_{1} y_{2} y_{1}=y_{2} y_{1} y_{2}$ and $y_{i}^{-1}=y_{i}^{2}$ we get

$$
y_{1}^{-1} y_{2} y_{1} y_{2}^{-1} y_{1}=y_{1}^{-1} y_{2} y_{1} y_{2} y_{2} y_{1}=y_{1}^{-1} y_{1} y_{2} y_{1} y_{2} y_{1}=y_{2} y_{1} y_{2} y_{1}=y_{2}^{-1} y_{1} y_{2} \text {. }
$$


It follows that

$$
\left(y_{11} \cdots y_{3}\right) y_{2}^{-1} y_{1} y_{2}\left(y_{11} \cdots y_{3}\right)^{-1}=\left(y_{2} \cdots y_{10}\right) y_{11}\left(y_{2} \cdots y_{10}\right)^{-1} \text {. }
$$

So $y_{1}$ can be expressed in terms of $y_{2}, \cdots, y_{11}$. So the reflections $\phi_{f_{2}}$ and $\phi_{f_{3}}$ can be expressed in terms of the 14 reflections stated in the lemma.

\section{The Fundamental Group of The MirRor COMPlEMENT-QUOTIENT}

In this section we shall show that the Artin group $\mathcal{A}(D)$ maps to the orbifold fundamental group of $Y^{\circ} / \Gamma$. So there is an action of $\mathcal{A}(D)$ on the universal cover of $Y^{\circ}$ and an action of $\operatorname{Cox}(D, 2)$ on a (possibly ramified) cover of $Y^{\circ} / \Gamma$.

4.1. Some basics on complex hyperbolic space for estimating distances. For this sub-section, we let $V$ be a general $(n+1)$ dimensional complex vector space with a Hermitian form of signature $(1, n)$. At the end of the sub-section we shall go back to our example. Let $V^{+}$be the set of vectors of strictly positive norm in $V$ and let $\mathbb{P}_{+}(V) \simeq \mathbb{C} H^{n}$ be the complex hyperbolic space of $V$. Whenever we talk of distance in this section, it is with reference to the metric on $\mathbb{C} H^{n}$. Given $x, y$ in $\mathbb{C} H^{n}$, let $\operatorname{Geod}(x, y)$ be the real geodesic segment joining $x$ and $y$.

In the following, let $x_{1}, \cdots, x_{n}, x, y, z, w$ be elements of $V^{+}$. Assume further that $x_{1}, \cdots, x_{n}$ lie in a totally real subspace, that is, $\left\langle x_{i}, x_{j}\right\rangle \in \mathbb{R}_{+}$for $1 \leq i, j \leq n$. Then, for all $i \neq j$ the Euclidean straight line segment in $V^{+}$joining $x_{i}$ and $x_{j}$ (denoted by $\operatorname{Conv}\left(x_{i}, x_{j}\right)$ ) determines the real geodesic segment in $\mathbb{C} H^{n}$ joining $x_{i}$ and $x_{j}$, that is, $\left[\operatorname{Conv}\left(x_{i}, x_{j}\right)\right]=\operatorname{Geod}\left(\left[x_{i}\right],\left[x_{j}\right]\right)$. Let $\operatorname{Conv}\left(x_{1}, \cdots, x_{n}\right)$ be the convex hull of $x_{1}, \cdots, x_{n}$ in $V^{+}$:

$$
\operatorname{Conv}\left(x_{1}, \cdots, x_{n}\right)=\left\{t_{1} x_{1}+\cdots+t_{n} x_{n}: 0 \leq t_{j} \leq 1, \sum t_{j}=1\right\} .
$$

The set $\operatorname{Conv}\left(x_{1}, \cdots, x_{n}\right)$ determines a totally real, totally geodesic subset of $\mathbb{C} H^{n}$. Given two non-empty subsets $Z$ and $W$ of $\mathbb{C} H^{n}$ with $W$ compact, let

$$
\operatorname{md}_{Z}(W)=\max \{d(Z, w): w \in W\} .
$$

The following observations will be useful for our computation. (Recall our assumption: $\left\langle x_{i}, x_{j}\right\rangle \in \mathbb{R}_{+}$.)

(1) Let $\left\{W_{j}: j \in J\right\}$ be a (possibly infinite) collection of compact sets such that $\bigcup_{j \in J} W_{j}$ is compact. Then

$$
\operatorname{md}_{H}\left(\bigcup_{j \in J} W_{j}\right)=\sup \left\{\operatorname{md}_{H}\left(W_{j}\right): j \in J\right\} .
$$

(2) By general properties of negatively curved spaces, one knows that $\max \left\{d(z, w): w \in \operatorname{Conv}\left(x_{1}, x_{2}\right)\right\}$ is attained when $w$ is either $x_{1}$ or $x_{2}$, in other words,

$$
\operatorname{md}_{z}\left(\operatorname{Conv}\left(x_{1}, x_{2}\right)\right)=\max \left\{d\left(z, x_{1}\right), d\left(z, x_{2}\right)\right\} .
$$

(3) Let $W=\operatorname{Conv}\left(x_{2}, \cdots, x_{n}\right)$. Using

$$
\operatorname{Conv}\left(x_{1}, x_{2}, \cdots, x_{n}\right)=\bigcup_{w \in W} \operatorname{Conv}\left(x_{1}, w\right)
$$

it follows that

$$
\begin{aligned}
\operatorname{md}_{z}\left(\operatorname{Conv}\left(x_{1}, \cdots, x_{n}\right)\right) & =\sup \left\{\operatorname{md}_{z}\left(\operatorname{Conv}\left(x_{1}, w\right)\right): w \in W\right\} \\
& =\max \left\{d\left(z, x_{1}\right), \operatorname{md}_{z}(W)\right\}
\end{aligned}
$$


By induction on $n$, it follows that $\max \left\{d(z, w): w \in \operatorname{Conv}\left(x_{1}, x_{2}, \cdots, x_{n}\right)\right\}$ is attained when $w=x_{i}$ for some $i$.

(4) Let $H$ be a subset of $\mathbb{C} H^{n}$ and $\Delta=\operatorname{Conv}\left(x_{1}, x_{2}, x_{3}\right)$. Let

$$
\delta_{1}=d\left(x_{1}, H\right)+\max \left\{d\left(x_{1}, x_{2}\right), d\left(x_{1}, x_{3}\right)\right\} .
$$

Define $\delta_{2}, \delta_{3}$ similarly by cyclic permutation of $x_{1}, x_{2}, x_{3}$. Choose $w_{0} \in \Delta$ such that $\operatorname{md}_{H}(\Delta)=d\left(w_{0}, H\right)$. Then, from the previous remarks, we have $\operatorname{md}_{H}(\Delta)=d\left(w_{0}, H\right) \leq d\left(x_{1}, H\right)+d\left(x_{1}, w_{0}\right) \leq d\left(x_{1}, H\right)+\operatorname{md}_{x_{1}}(\Delta) \leq \delta_{1}$.

It follows that

$$
\operatorname{md}_{H}(\Delta) \leq \min \left\{\delta_{1}, \delta_{2}, \delta_{3}\right\} .
$$

(5) Let $x \in V^{+}$and $H$ be a complex linear subspace in $V$ which meets $V^{+}$. Then $H$ determines a totally geodesic subspace of $\mathbb{C} H^{n}$. Let $\operatorname{pr}_{H}(x)$ be the "projection" of $[x]$ on $H$, that is, the point on $[H]$ that is closest to $[x]$.

Let $r_{1}, \cdots, r_{k} \in V$ be linearly independent vectors of negative norm and let $H=r_{1}^{\perp} \cap \cdots \cap r_{k}^{\perp}$. Assume that $H \cap V^{+} \neq \emptyset$; in other words, the span of $\left\{r_{1}, \cdots, r_{k}\right\}$ is negative definite. Then $[H]$ determines a totally geodesic subspace of $\mathbb{C} H^{n}$, and one has

$$
\operatorname{pr}_{H}(x)=\left(x+\mathbb{C} r_{1}+\cdots+\mathbb{C} r_{k}\right) \cap H .
$$

4.2. The setup. Let $L$ be the lattice already encountered in the introduction and section 3 (the direct sum of the complex Leech lattice and a hyperbolic cell). Let $Y=\mathbb{P}_{+}\left(L^{\mathbb{C}}\right) \simeq \mathbb{C} H^{13}$ and $\mathcal{M}$ be the union of the mirrors of the reflection group of $L$. Let $Y^{\circ}=Y \backslash \mathcal{M}$ be the complement of the mirrors and let $X^{\circ}=Y^{\circ} / \Gamma$. A continuous function $\gamma:[0,1] \rightarrow Y^{\circ}$ will be called a path in $Y^{\circ}$. Let $\Pi_{Y^{\circ}}(a, b)$ be the set of homotopy class of paths (rel. endpoints) in $Y^{\circ}$ beginning at $a$ and ending at $b$. Given two paths $\gamma$ and $\gamma^{\prime}$ in $Y^{\circ}$ with $\gamma(1)=\gamma^{\prime}(0)$, let $\gamma * \gamma^{\prime}$ be the path obtained by first following $\gamma$ and then following $\gamma^{\prime}$ at double the speed. Let $\gamma_{1}$ and $\gamma_{2}$ be two paths in $Y^{\circ}$ with the same beginning and end points. If $\gamma_{1}$ and $\gamma_{2}$ are homotopic rel. the endpoint in $Y^{\circ}$, then we write $\gamma_{1} \sim \gamma_{2}$. The homotopy class of a path $\gamma$ is denoted by $[\gamma]$.

Definition 4.3. Let $G=\left\{(\gamma, t): t \in \Gamma, \gamma \in \Pi_{Y^{\circ}}(\bar{\rho}, t \bar{\rho})\right\}$. Then $G$ becomes a group with multiplication defined by

$$
(\gamma, t) \cdot\left(\gamma^{\prime}, t^{\prime}\right)=\left(\gamma * t \gamma^{\prime}, t t^{\prime}\right)
$$

Define $\pi_{\Gamma}^{G}: G \rightarrow \Gamma$ by $\pi_{\Gamma}^{G}(\gamma, t)=t$. The kernel of the epimorphism $\pi_{\Gamma}^{G}$ is $\pi_{1}\left(Y^{\circ}, \bar{\rho}\right)$. So we have an exact sequence

$$
1 \rightarrow \pi_{1}\left(Y^{\circ}\right) \rightarrow G \stackrel{\pi_{\Gamma}^{G}}{\longrightarrow} \Gamma \rightarrow 1 .
$$

Let $\tilde{Y}^{\circ}=\bigcup_{y \in Y^{\circ}} \Pi_{Y^{\circ}}(\bar{\rho}, y)$ be the universal cover of $Y^{\circ}$. An element of $\tilde{Y}^{\circ}$ lying above $y \in Y^{\circ}$ is represented by a path $\lambda:[0,1] \rightarrow Y^{\circ}$ such that $\lambda(1)=y$. The group $G$ acts on $\tilde{Y}^{\circ}$ by

$$
([\gamma], t)[\lambda]=[\gamma * t \lambda]
$$

Let $\pi_{X^{\circ}}^{\tilde{Y}^{\circ}}: \tilde{Y}^{\circ} \rightarrow X^{\circ}$ be the projection given by $[\lambda] \mapsto \Gamma \lambda(1)$. Then $\pi_{X^{\circ}}^{\tilde{Y}^{\circ}}(g[\lambda])=$ $\pi_{X^{\circ}}^{\tilde{Y}^{\circ}}([\lambda])$ for all $g \in G$; that is, $G$ acts as deck transformations on the ramified covering $\tilde{Y}^{\circ} \rightarrow X^{\circ}$. For the purpose of this article, we call $G$ the orbifold fundamental group of $X^{\circ}$. 


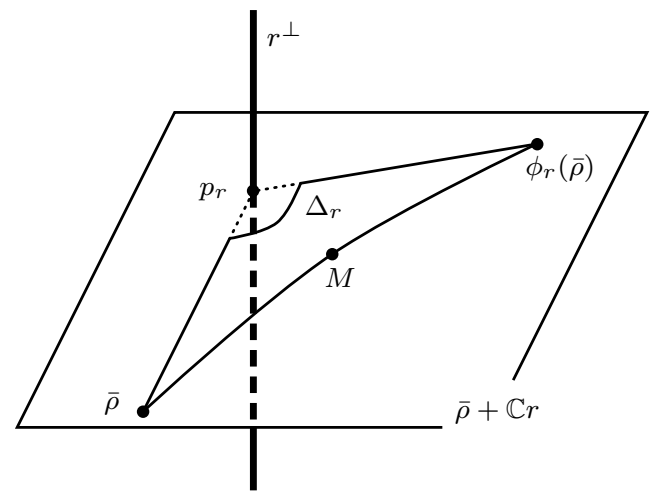

Figure 1. A totally geodesic triangle $\Delta_{r}$ such that $\Delta_{r} \backslash\left\{p_{r}\right\}$ does not intersect any mirror.

By the results mentioned in section 3, there is a surjection $\phi: \mathcal{A}(D) \rightarrow \Gamma$ taking the generators of $\mathcal{A}(D)$ to order 3 simple reflections in $\Gamma$. We want to prove the following theorem.

Theorem 4.4. There exists a homomorphism $\psi: \mathcal{A}(D) \rightarrow G$ such that $\pi_{\Gamma}^{G} \circ \psi=\phi$.

To give a flavor of the argument, we first prove an easy model lemma.

Lemma 4.5. (a) Let $r$ be a simple root of L. Let $p_{r}$ be the projection of $\bar{\rho}$ on $r^{\perp}$. Let $\Delta_{r}$ be the closed totally geodesic triangle in $Y$ with vertices at $\bar{\rho}, p_{r}=\operatorname{pr}_{r^{\perp}}(\bar{\rho})$ and $\phi_{r}(\bar{\rho})$ (see Figure 1). Then $\Delta_{r} \backslash\left\{p_{r}\right\}$ does not intersect any mirror.

(b) Let $\gamma$ and $\gamma^{\prime}$ be any two paths lying in $\Delta_{r} \backslash\left\{p_{r}\right\}$, starting at $\bar{\rho}$ and ending at $\phi_{x}(\bar{\rho})$. Then $\gamma \sim \gamma^{\prime}$ in $Y^{\circ}$.

Proof. The isosceles triangle $\Delta_{r}$ is totally geodesic, since it lies in the complex geodesic $\mathbb{P}_{+}(\mathbb{C} r+\mathbb{C} \bar{\rho})$, containing $\bar{\rho}$ and $r$. Let $M$ be the midpoint of $\bar{\rho}$ and $\phi_{r}(\bar{\rho})$. Let $\Delta_{r}^{1}$ be the triangle with vertices $\bar{\rho}, p_{r}, M$ and let $\Delta_{r}^{2}$ be the triangle with vertices $\phi_{r}(\bar{\rho}), p_{r}, M$. So $\Delta_{r}=\Delta_{r}^{1} \cup \Delta_{r}^{2}$. One checks that $d\left(\bar{\rho}, p_{r}\right)>d(\bar{\rho}, M)$. So the point of $\Delta_{r}^{1}$ that is furthest from $\bar{\rho}$ is $p_{r}$. But $d\left(\bar{\rho}, p_{r}\right)$ is the minimum distance between $\bar{\rho}$ and any mirror (by Theorem $1.2(\mathrm{c})$ ). Hence no mirror can intersect $\Delta_{r}^{1} \backslash\left\{p_{r}\right\}$. The same statement holds for $\Delta_{r}^{2}$ by symmetry. This proves part (a). Part (b) follows from part (a).

Definition 4.6. Maintain the notation of Lemma 4.5, Let $r$ be a simple root. Let $[\gamma]_{r}$ be the unique homotopy class of paths lying in $\Delta \backslash\left\{p_{r}\right\}$ starting at $\bar{\rho}$ and ending at $\phi_{r}(\bar{\rho})$. Define $g_{r} \in G$, called the braid reflection in $r$, by $g_{r}=\left([\gamma]_{r}, \phi_{r}^{\omega}\right)$. If $r_{1}, r_{2}, \cdots, r_{26}$ are the simple roots, then we write $\phi_{i}=\phi_{r_{i}}^{\omega},[\gamma]_{i}=[\gamma]_{r_{i}}$ and $g_{i}=g_{r_{i}}$.

Now, Theorem 4.4 follows from the following result.

Theorem 4.7. Let $r_{1}$ and $r_{2}$ be two simple roots of $L$. If the $\omega$-reflections $\phi_{1}$ and $\phi_{2}$ braid (resp. commute) in $\Gamma$, then the braid reflections $g_{1}$ and $g_{2}$ braid (resp. commute) in $G$. So $\psi\left(r_{i}\right)=g_{i}$ defines a homomorphism $\psi: \mathcal{A}(D) \rightarrow G$ such that $\pi_{\Gamma}^{G} \circ \psi=\phi$.

The rest of this section is devoted to proving Theorem 4.7. First we describe a sketch of the proof that states some lemmas along the way. Then we give detailed proofs of the lemmas. 


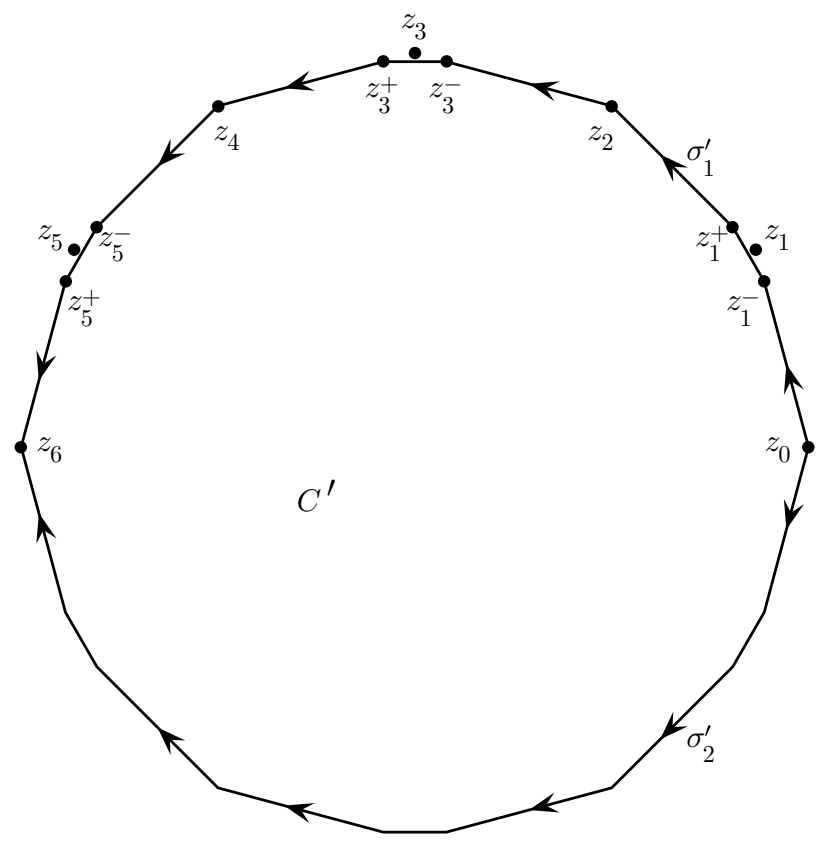

Figure 2. Schematic picture of the 2-cell $C^{\prime}$ and the boundary curves $\sigma_{1}^{\prime}$ and $\sigma_{2}^{\prime}$. The points $z_{k}$ and $z_{2 j-1}^{ \pm}$on $\sigma_{1}^{\prime}$ are marked.

Sketch of proof. We need to set up some notation to make our way smooth.

Let $r_{1}, \cdots, r_{26}$ be the simple roots. Define the vectors $D_{\rho}=\left\{\rho_{1}, \cdots, \rho_{26}\right\}$ by

$$
\rho_{j}= \begin{cases}r_{j} & \text { if } r_{j} \in \mathcal{P} \\ \xi r_{j} & \text { if } r_{j} \in \mathcal{L} .\end{cases}
$$

Sometimes it is more convenient to use the vectors $\rho_{j}$ instead of $r_{j}$. Recall that for all $i$ and $j,\left\langle\rho_{i}, \rho_{j}\right\rangle$ is a non-negative real number, $\bar{\rho}=\sum_{i=1}^{26} \rho_{i} / 26$ and $\left\langle\rho_{i}, \bar{\rho}\right\rangle=|\bar{\rho}|^{2}$.

Let $r_{1}$ and $r_{2}$ be any two distinct simple roots and let $\rho_{1}$ and $\rho_{2}$ be the corresponding elements of $D_{\rho}$. Let

$$
p_{i}=\bar{\rho}+\frac{|\bar{\rho}|^{2}}{3} \rho_{i} \quad \text { and } \quad q=\bar{\rho}+\frac{|\bar{\rho}|^{2}}{\alpha}\left(\rho_{1}+\rho_{2}\right), \quad \text { where } \alpha= \begin{cases}3 & \text { if }\left\langle\rho_{1}, \rho_{2}\right\rangle=0 \\ 3-\sqrt{3} & \text { if }\left\langle\rho_{1}, \rho_{2}\right\rangle=\sqrt{3}\end{cases}
$$

Then $\left[p_{1}\right],\left[p_{2}\right]$ and $[q]$ are the projections of $\bar{\rho}$ on $\rho_{1}^{\perp}, \rho_{2}^{\perp}$ and $\rho_{1}^{\perp} \cap \rho_{2}^{\perp}$ respectively.

Suppose the reflections in $r_{1}$ and $r_{2}$ braid. Let $\phi_{1}=\phi_{r_{1}}^{\omega}$ and $\phi_{2}=\phi_{r_{2}}^{\omega}$. We define vectors $z_{0}, \cdots, z_{6}$ as follows (see Figure 2):

$$
\begin{gathered}
z_{0}=\bar{\rho}, \quad z_{1}=p_{1}, \quad z_{2}=\phi_{1}(\bar{\rho}), \quad z_{3}=\phi_{1}\left(p_{2}\right), \\
z_{4}=\phi_{1} \phi_{2}(\bar{\rho}), \quad z_{5}=\phi_{1} \phi_{2}\left(p_{1}\right), \quad z_{6}=\phi_{2} \phi_{1} \phi_{2}(\bar{\rho}) .
\end{gathered}
$$

Recall that $\left\langle\rho_{i}, \rho\right\rangle=|\rho|^{2}$ and $\left\langle\rho_{i}, \rho_{j}\right\rangle$ are all non-negative real numbers. Using these, one checks that $\left\langle z_{j-1}, z_{j}\right\rangle \in \mathbb{R}_{+}$, for $j=1, \cdots, 6$ and $\left\langle z_{j}, q\right\rangle \in \mathbb{R}_{+}$for $j=0,1, \cdots, 6$. In particular, the geodesic segment joining $z_{j-1}$ and $z_{j}$ in $Y$ follows 
the curve $\operatorname{Conv}\left(z_{j-1}, z_{j}\right)$. We shall use complex hyperbolic geometry to prove the following lemma.

Lemma 4.8. Let $\sigma_{1}=\bigcup_{j=1}^{6} \operatorname{Conv}\left(z_{j-1}, z_{j}\right)$. Define $\sigma_{2}$ similarly by interchanging the roles of $r_{1}$ and $r_{2}$. Let $C=\bigcup_{x \in \sigma_{1} \cup \sigma_{2}} \operatorname{Conv}(q, x) \subseteq V_{+}$. Then the 2 -cell $C$ only intersects the four mirrors $r_{1}^{\perp}, r_{2}^{\perp}, \phi_{r_{1}}\left(r_{2}\right)^{\perp}$ and $\phi_{r_{2}}\left(r_{1}\right)^{\perp}$.

Note that $\bigcup_{x \in \sigma_{1}} \operatorname{Conv}(q, x)=\bigcup_{j=1}^{6} \operatorname{Conv}\left(q, z_{j-1}, z_{j}\right)$ and $\bigcup_{x \in \sigma_{2}} \operatorname{Conv}(q, x)$ is a union of six similar totally geodesic triangles and $C$ is the union of these. Note that $\sigma_{1}$ and $\sigma_{2}$ are curves in $Y$ (not in $Y^{\circ}$ ). The curve $\sigma_{1}$ contains the points $z_{1}$, $z_{3}$ and $z_{5}$ which lie on mirrors. So we need to modify $\sigma_{1}$ to avoid this points. For this, fix a small positive real number $\epsilon$. For $j=1,2,3$, let

$$
z_{2 j-1}^{-}=z_{2 j-1}+\epsilon z_{2 j-2} \text { and } z_{2 j-1}^{+}=z_{2 j-1}+\epsilon z_{2 j}
$$

and let $\tilde{\gamma}_{j}$ be a path in $V_{+}$that goes from $z_{2 j-2}$ to $z_{2 j}$ along the curve

$$
\operatorname{Conv}\left(z_{2 j-2}, z_{2 j-1}^{-}\right) \cup \operatorname{Conv}\left(z_{2 j-1}^{-}, z_{2 j-1}^{+}\right) \cup \operatorname{Conv}\left(z_{2 j-1}^{+}, z_{2 j}\right) \text {. }
$$

Define $\sigma_{1}^{\prime}=\tilde{\gamma}_{1} * \tilde{\gamma}_{2} * \tilde{\gamma}_{3}$ (see Figure 2). Similarly define $\sigma_{2}^{\prime}$ by interchanging the role of $r_{1}$ and $r_{2}$. Lemma 4.5 implies that $\left[\tilde{\gamma}_{1}\right]=[\gamma]_{1}$. Similarly, $\left[\tilde{\gamma}_{2}\right]=\phi_{1}[\gamma]_{2}$ and $\left[\tilde{\gamma}_{3}\right]=\phi_{1} \phi_{2}[\gamma]_{1}$. So $g_{1} g_{2} g_{1}=\left(\left[\sigma_{1}^{\prime}\right], t\right)$ where $t=\phi_{1} \phi_{2} \phi_{1}=\phi_{2} \phi_{1} \phi_{2}$. Similarly $g_{2} g_{1} g_{2}=\left(\left[\sigma_{2}^{\prime}\right], t\right)$. We need to prove that $\sigma_{1}^{\prime} \sim \sigma_{2}^{\prime}$. This follows from the lemma given below.

Lemma 4.9. Let $q^{\prime}=q+\epsilon \bar{\rho}$. Then the 2 -cell $C^{\prime}=\bigcup_{x \in \sigma_{1}^{\prime} \cup \sigma_{2}^{\prime}} \operatorname{Conv}\left(q^{\prime}, x\right) \subseteq V_{+}$ does not intersect any mirrors.

Given Lemma 4.8, the proof of Lemma 4.9 is easy. Since $C^{\prime}$ is a small perturbation of $C$, it suffices to check that the four mirrors intersecting $C$ do not intersect $C^{\prime}$. This can be checked by a direct calculation that is given at the end of this section. This completes the argument in the case when $\phi_{1}$ and $\phi_{2}$ braid. When $\phi_{1}$ and $\phi_{2}$ commute, the argument is similar and the calculations are simpler. We briefly indicate the modifications that are needed.

Assume that $\phi_{1}$ and $\phi_{2}$ commute. Let

$$
z_{0}=\bar{\rho}, \quad z_{1}=p_{1}, \quad z_{2}=\phi_{1}(\bar{\rho}), \quad z_{3}=\phi_{1}\left(p_{2}\right), \quad z_{4}=\phi_{1} \phi_{2}(\bar{\rho}) .
$$

Instead of Lemma 4.8 we have the following lemma:

Lemma 4.10. Let $\sigma_{1}=\bigcup_{j=1}^{4} \operatorname{Conv}\left(z_{j-1}, z_{j}\right)$. Define $\sigma_{2}$ similarly by interchanging the roles of $r_{1}$ and $r_{2}$. Then the 2 -cell $C=\bigcup_{x \in \sigma_{1} \cup \sigma_{2}} \operatorname{Conv}(q, x)$ only intersects the mirrors $r_{1}^{\perp}$ and $r_{2}^{\perp}$.

Define $z_{2 j-1}^{ \pm}$and $\tilde{\gamma}_{j}$ for $j=1,2$ by the formulas given in (11) and (12). Let $\sigma_{1}^{\prime}=\tilde{\gamma}_{1} * \tilde{\gamma}_{2}$. Similarly define $\sigma_{2}^{\prime}$. With this setting, one has to re-prove Lemma 4.9, which amounts to checking that $r_{1}^{\perp}$ and $r_{2}^{\perp}$ do not intersect $C^{\prime}$.

It remains to prove the lemmas stated in the sketch above.

Proof of Lemmas 4.8 and 4.10. Let $p_{1}, p_{2}$ and $q$ be as given in (10). Note that the inner products between $\bar{\rho}, p_{1}, p_{2}$ and $q$ are all real and they lie in a totally geodesic subspace $P$ of $\mathbb{C} H^{13}$ isomorphic to the real hyperbolic plane. The 2-cell $C \subseteq V^{+}$ given in Lemma 4.8 (resp. Lemma 4.10) is a union of 12 (resp. 8) Euclidean triangles, and $[C]$ is a union of 12 (resp. 8) totally geodesic triangles in $Y$ (because 
$q$ has real inner product with the vertices of $\sigma_{1}$ and $\sigma_{2}$ ). The boundary of $C$ is $\sigma_{1} \cup \sigma_{2}$.

Consider the quadrilateral $T=\operatorname{Conv}\left(\bar{\rho}, p_{1}, q\right) \cup \operatorname{Conv}\left(\bar{\rho}, p_{2}, q\right)$. If $\phi_{1}$ and $\phi_{2}$ braid, then, with $C$ as given in Lemma 4.8, we have

$$
C=T \cup \phi_{1}(T) \cup \phi_{2}(T) \cup \phi_{1} \phi_{2}(T) \cup \phi_{2} \phi_{1}(T) \cup \phi_{1} \phi_{2} \phi_{1}(T) .
$$

If $\phi_{1}$ and $\phi_{2}$ commute, then, with $C$ as given in Lemma 4.10, we have

$$
C=T \cup \phi_{1}(T) \cup \phi_{2}(T) \cup \phi_{1} \phi_{2}(T) .
$$

There is a diagram automorphism that fixes $\bar{\rho}$ and interchanges $\rho_{1}$ and $\rho_{2}$, so the intersection of the triangle $\operatorname{Conv}\left(\bar{\rho}, p_{2}, q\right)$ with the mirrors is similar to that of the triangle $\operatorname{Conv}\left(\bar{\rho}, p_{1}, q\right)$. Lemmas 4.8 and 4.10 now follow from Lemma 4.11, given below.

Lemma 4.11. Let $\bar{\rho}, \rho_{1}, \rho_{2}, p_{1}, p_{2}, q$ be as introduced in the sketch of the proof of Theorem 4.7. The triangle $\operatorname{Conv}\left(\bar{\rho}, p_{1}, q\right)$ meets the mirror $\rho_{1}^{\perp}$ along the edge $\operatorname{Conv}\left(p_{1}, q\right)$, meets $\rho_{2}^{\perp}$ at $q$ and, in the case when $\left\langle\rho_{1}, \rho_{2}\right\rangle=\sqrt{3}$, also meets $\phi_{\rho_{1}}^{ \pm}\left(\rho_{2}\right)^{\perp}$ at $q$. Except for these cases no other mirrors intersect $\operatorname{Conv}\left(\bar{\rho}, p_{1}, q\right) \backslash\left\{p_{1}\right\}$.

Proof. The proof given below uses some computer verification. These calculations were performed using the GP/PARI calculator. The codes are contained in the file pil.gp available at https://orion.math.iastate.edu/tathagat/codes/ index.html.

The diagonal action of $\operatorname{PGL}\left(3, \mathbb{F}_{3}\right) .2$ on distinct pairs of simple roots has three orbits:

(1) $\rho_{1} \in \mathcal{P}, \bar{\xi} \rho_{2} \in \mathcal{L}$ and $\left\langle\rho_{1}, \rho_{2}\right\rangle=\sqrt{3}$; for calculation we take $\rho_{1}=a, \rho_{2}=\xi f$.

(2) $\rho_{1} \in \mathcal{P}, \bar{\xi} \rho_{2} \in \mathcal{L}$ and $\left\langle\rho_{1}, \rho_{2}\right\rangle=0$; for calculation we take $\rho_{1}=a, \rho_{2}=\xi d_{1}$.

(3) $\rho_{1} \in \mathcal{P}$ and $\rho_{2} \in \mathcal{P}$; for calculation we take $\rho_{1}=a, \rho_{2}=c_{1}$.

Accordingly, we have to consider three cases in the calculations below. If not stated otherwise, the statements below are made for all three cases. It will be convenient to define the height of a root $r$, denoted by ht $(r)$, as follows:

$$
\tilde{\mathrm{ht}}(r)=\langle\bar{\rho}, r\rangle /|\bar{\rho}|^{2} \quad \text { and } \operatorname{ht}(r)=|\tilde{\mathrm{ht}}(r)| .
$$

Let $d_{0}=d\left(\bar{\rho}, p_{1}\right)$. This is the minimum distance from $\bar{\rho}$ to any mirror. Suppose $r^{\perp}$ is a mirror that intersects the triangle $\Delta_{1}=\operatorname{Conv}\left(\bar{\rho}, p_{1}, q\right)$. The longest edge of the triangle $\Delta_{1}$ is $\operatorname{Conv}(\bar{\rho}, q)$. So

$$
\sinh ^{-1}\left(\frac{|\langle r, \bar{\rho}\rangle|}{|r||\bar{\rho}|}\right)=d\left(r^{\perp}, \bar{\rho}\right) \leq \operatorname{md}_{\bar{\rho}}\left(\Delta_{1}\right)=d(\bar{\rho}, q) .
$$

This gives a bound for the possible height of the root $r$ :

$$
\operatorname{ht}(r)=\frac{|\langle r, \bar{\rho}\rangle|}{|\bar{\rho}|^{2}} \leq \frac{|r|}{|\bar{\rho}|} \sinh \left(\cosh ^{-1}\left(\frac{|\langle\bar{\rho}, q\rangle|}{|\bar{\rho}||q|}\right)\right) \leq 2.18 .
$$

Let $s$ be the point on $\operatorname{Conv}(\bar{\rho}, q)$ such that $d(\bar{\rho}, s)=d_{0}$. The triangle $\operatorname{Conv}\left(\bar{\rho}, p_{1}, s\right)$ cannot meet any mirror except at $p_{1}$ and possibly at $s$. So the mirror $r^{\perp}$ must intersect the triangle $\Delta=\operatorname{Conv}\left(p_{1}, q, s\right)$. The situation is depicted in Figure 3 , One can take $s=\bar{\rho}+c\left(\rho_{1}+\rho_{2}\right)$ where $c \in \mathbb{R}_{+}$is a constant to be determined. The equality $d(s, \bar{\rho})=d_{0}$ implies

$$
1+\frac{|\bar{\rho}|^{2}}{3}=\frac{\left|\left\langle\bar{\rho}, p_{1}\right\rangle\right|^{2}}{|\bar{\rho}|^{2}\left|p_{1}\right|^{2}}=\frac{|\langle s, \bar{\rho}\rangle|^{2}}{|s|^{2}|\bar{\rho}|^{2}}=\frac{|\bar{\rho}|^{4}(1+2 c)^{2}}{\left(|\bar{\rho}|^{2}+4 c|\bar{\rho}|^{2}-2 \alpha c^{2}\right)|\bar{\rho}|^{2}}
$$




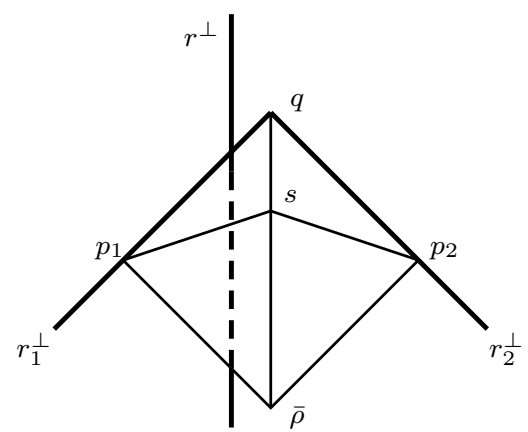

FiguRE 3

(where $\alpha$ is given in (10)). Re-arranging, one has the quadratic equation

$$
\left(4+\frac{2 \alpha}{|\bar{\rho}|^{2}}\left(1+\frac{|\bar{\rho}|^{2}}{3}\right)\right) c^{2}-\frac{4}{3}|\bar{\rho}|^{2} c-\frac{|\bar{\rho}|^{2}}{3}=0
$$

with one positive and one negative root. The positive root gives the required $c$.

Divide the triangle $\Delta$ into two triangles $\Delta^{\prime}$ and $\Delta^{\prime \prime}$ by joining $s$ with the midpoint of $\operatorname{Conv}\left(p_{1}, q\right)$. For $i=1, \cdots, 26$, one has

$$
d\left(\rho_{i}^{\perp}, r^{\perp}\right) \leq \operatorname{md}_{\rho_{i}^{\perp}}(\Delta)=\max \left\{\operatorname{md}_{\rho_{i}^{\perp}}\left(\Delta^{\prime}\right), \operatorname{md}_{\rho_{i}^{\perp}}\left(\Delta^{\prime \prime}\right)\right\},
$$

where the equality is an instance of equation (8) in section 4.1. We estimate $\operatorname{md}_{\rho_{i}^{\perp}}\left(\Delta^{\prime}\right)$ and $\operatorname{md}_{\rho_{i}^{\perp}}\left(\Delta^{\prime \prime}\right)$ using the inequality (9) of section 4.1. This gives a bound on $\left|\left\langle\rho_{i}, r\right\rangle\right|^{2}=9 \cosh ^{2}\left(d\left(\rho_{i}^{\perp}, r^{\perp}\right)\right)$. By explicit computation, this bound is strictly less than 12 except in three cases when $\left\langle\rho_{1}, \rho_{2}\right\rangle=\sqrt{3}$ and $\bar{\xi} \rho_{i} \in \mathcal{L}$. Since the inner product between two vectors of $L$ always lies in $p \mathcal{E}$ one has

$$
\left|\left\langle x_{i}, r\right\rangle\right|^{2} \in\{0,3,9\} \quad \text { for } i=1, \cdots, 13 .
$$

Similarly, from the inequality

$$
d\left(w_{\mathcal{P}}, r^{\perp}\right) \leq \operatorname{md}_{w_{\mathcal{P}}}(\Delta) \leq \max \left\{d\left(w_{\mathcal{P}}, p_{1}\right), d\left(w_{\mathcal{P}}, q\right), d\left(w_{\mathcal{P}}, s\right)\right\},
$$

one gets a bound on $\left|\left\langle w_{p}, r\right\rangle\right|^{2}=9 \sinh ^{2}\left(d\left(r^{\perp}, w_{\mathcal{P}}\right)\right)$. By explicit computation the bound is strictly less than 10 . So one has

$$
\left|\left\langle w_{\mathcal{P}}, r\right\rangle\right|^{2} \in\{0,3,9\} .
$$

The conditions (14) and (15) restrict the possibilities for $r$ to a finite set as in the proof of Proposition 6.1 in [5]. Write $r$ in terms of the orthogonal basis $\left(w_{\mathcal{P}}, x_{1}, \cdots, x_{13}\right)$ as

$$
r=\left\langle w_{\mathcal{P}}, r\right\rangle w_{\mathcal{P}} / 3-\sum\left\langle x_{i}, r\right\rangle x_{i} / 3
$$

Taking norm and re-arranging one gets

$$
\sum\left|\left\langle x_{i}, r\right\rangle\right|^{2}=9+\left|\left\langle w_{\mathcal{P}}, r\right\rangle\right|^{2},
$$

which leaves only finitely many possibilities for the vector $\left(\left\langle w_{\mathcal{P}}, r\right\rangle,\left\langle x_{1}, r\right\rangle, \cdots\right.$, $\left.\left\langle x_{13}, r\right\rangle\right)$. The possible inner products of $r$ with $x_{i}$ and $w_{p}$ are shown in Table 1, By inspection of the last three entries of the fifth column, we find that the minimum height of a root having type 5,6 or 7 is greater than the bound on ht $(r)$ obtained 
TABLE 1. In each row, we consider roots $r$ of a certain form, determined by the possible inner products of $r$ with $w_{\mathcal{P}}$ and with $x_{1}, \cdots, x_{13}$ as shown in the second and third columns respectively. $\left(u_{1}, u_{2}, \cdots\right.$ stand for sixth roots of unity.) The roots considered in the $j$-th row are called the roots of type $j$. The fourth column records $\tilde{\mathrm{ht}}(r)=\langle\bar{\rho}, r\rangle /|\bar{\rho}|^{2}$ for a root $r$ of type $j$. The fifth column records the minimum possible absolute value of the entry in the fourth column as $u_{i}$ 's vary over the sixth roots of unity, that is, the minimum possible height of a root of type $j$.

\begin{tabular}{|c|c|c|c|c|}
\hline$j$ & $\left\langle w_{\mathcal{P}}, r\right\rangle$ & possible $\left(\left\langle x_{1}, r\right\rangle, \cdots,\left\langle x_{13}, r\right\rangle\right)$ & $\tilde{\mathrm{ht}}(r)$ & $\min (\mathrm{ht}(r))$ \\
\hline 1 & 0 & $\left(3 u_{1}, 0^{12}\right)$ & $-u_{1}$ & 1 \\
2 & & $\theta\left(u_{1}, u_{2}, u_{3}, 0^{10}\right)$ & $\frac{1}{\theta} \sum_{i=1}^{3} u_{i}$ & no root \\
\hline 3 & $\theta$ & $\left(3 u_{1}, \theta u_{2}, 0^{11}\right)$ & $\frac{1}{\theta}\left(-4-\sqrt{3}+u_{1}-\theta u_{2}\right)$ & no root \\
4 & & $\theta\left(u_{1}, u_{2}, u_{3}, u_{4}, 0^{9}\right)$ & $\frac{1}{\theta}\left(-4-\sqrt{3}+\sum_{i=1}^{4} u_{i}\right)$ & 1 \\
\hline 5 & 3 & $3\left(u_{1}, u_{2}, 0^{11}\right)$ & $4+\sqrt{3}-u_{1}-u_{2}$ & $2+\sqrt{3}$ \\
6 & & $\left(3 u_{1}, \theta u_{2}, \theta u_{3}, \theta u_{4}, 0^{9}\right)$ & $4+\sqrt{3}-u_{1}+\frac{1}{\theta} \sum_{i=2}^{4} u_{i}$ & 3.24 \\
7 & & $\theta\left(u_{1}, \cdots, u_{6}, 0^{7}\right)$ & $4+\sqrt{3}+\frac{1}{\theta} \sum_{i=1}^{6} u_{i}$ & 2.73 \\
\hline
\end{tabular}

in (13). So mirrors of type 5,6 or 7 cannot intersect $\Delta_{1}$. Next, one checks that there are no roots of type 2 or 3 . This implies $r$ must be of type 1 or 4 . The only mirrors of type 1 are the 13 simple mirrors corresponding to $\mathcal{P}$. Now we make a list of those type 4 roots that satisfy ht $(r)<2.2$ (which is enough in view of inequality (13)). The list consists of the 13 simple mirrors corresponding to $\mathcal{L}$ and the 104 mirrors corresponding to the roots of the form $\phi_{x}^{ \pm}(l)$, where $x \in \mathcal{P}, y \in \mathcal{L}$ and $x$ is incident on $l$. (In fact these are the only roots having height $|1+\xi|$, but we shall not use this fact.) We want to show that these 130 mirrors do not meet $\Delta_{1}$ except for the cases described in the statement of Lemma 4.11. This is checked on the computer as follows:

Let $P=\left\{\left[\bar{\rho}+s_{2} p_{1}+s_{3} q\right]: s_{j} \in \mathbb{R}\right\} \subseteq \mathbb{P}(V)$. Then $P$ contains the triangle $\Delta_{1}$. If the complex numbers $\langle r, \bar{\rho}\rangle,\left\langle r, p_{1}\right\rangle$ and $\langle r, q\rangle$ all have the same argument, then we find that $\operatorname{Re}(\langle r, \bar{\rho}\rangle)=\operatorname{Re}\left(\left\langle r, p_{1}\right\rangle\right)=\operatorname{Re}(\langle r, q\rangle)=|\bar{\rho}|^{2}$. So a convex combination of $\bar{\rho}, p_{1}$ and $q$ cannot be orthogonal to $r$. Otherwise $r^{\perp} \cap P$ is a point in $\mathbb{P}(V)$ which can be found by solving two linear equations. We have checked that this point does not belong to $\Delta_{1}$ except in the cases mentioned in the statement of the lemma. 
TABLE 2. The left and top margin gives the row and column numbers (denoted by $j$ and $k$ ). The $j$-th row of right margin records $r_{j}$. The $k$-th column in the bottom margin records $z_{k}$. The entry in the $j$-th row and $k$-th column is $c_{j}^{k}=\left\langle z_{k}, r_{j}\right\rangle /|\bar{\rho}|^{2}$. Finally, $c=$ $1+3^{-1 / 2}$.

\begin{tabular}{c|ccccccc|c}
$j \backslash k$ & 0 & 1 & 2 & 3 & 4 & 5 & 6 & $r_{j}$ \\
\hline 1 & 1 & 0 & $\bar{\omega}$ & $\bar{\omega} c$ & $\bar{\omega}-i$ & $-i c$ & $-i$ & $r_{1}$ \\
2 & $\bar{\xi}$ & $\bar{\xi} c$ & $\bar{\xi}-\bar{\omega}$ & $-\bar{\omega} c$ & $-\bar{\omega}$ & 0 & $-\omega$ & $r_{2}$ \\
3 & $1+\bar{\xi}$ & $\bar{\xi} c$ & $\bar{\xi}$ & 0 & $-i$ & $-i c$ & $-i-\omega$ & $r_{1}+r_{2}$ \\
4 & $1+\xi$ & $\xi c$ & $\xi-\omega$ & $-\omega c$ & $-\omega-i$ & $-i c$ & $-i+\bar{\omega}$ & $r_{1}-\omega r_{2}$ \\
\hline$z_{k}$ & $\bar{\rho}$ & $p_{1}$ & $\phi_{x}(\bar{\rho})$ & $\phi_{x}\left(p_{2}\right)$ & $\phi_{x} \phi_{l}(\bar{\rho})$ & $\phi_{x} \phi_{l}\left(p_{1}\right)$ & $\phi_{l} \phi_{x} \phi_{l}(\bar{\rho})$ &
\end{tabular}

Proof of Lemma 4.9. Assume that $r_{1} \in \mathcal{P}, r_{2} \in \mathcal{L}$ and that $\phi_{r_{1}}$ and $\phi_{r_{2}}$ braid. Let $r_{3}=\phi_{r_{1}}\left(r_{2}\right)=\phi_{r_{2}}^{-1}\left(r_{1}\right)=r_{2}+r_{1}$ and $r_{4}=\phi_{r_{2}}\left(r_{1}\right)=\phi_{r_{1}}^{-1}\left(-\omega r_{2}\right)=r_{1}-\omega r_{2}$.

It suffices to show that the mirrors $\left\{r_{1}^{\perp}, \cdots, r_{4}^{\perp}\right\}$ do not meet $C^{\prime}$. For this, it is enough to show that $\bigcup_{x \in \sigma_{1}^{\prime}} \operatorname{Conv}\left(q^{\prime}, x\right)$ do not intersect these four mirrors, since $\bigcup_{x \in \sigma_{2}^{\prime}} \operatorname{Conv}\left(q^{\prime}, x\right)$ can then be handled by symmetry, as we explain in the rest of this paragraph. Note that there exists a diagram automorphism in $Q \simeq \operatorname{PGL}\left(3, \mathbb{F}_{3}\right) .2$ that takes $\left(\rho_{1}, \rho_{2}, \bar{\rho}\right)$ to $\left(\xi \rho_{2}, \xi \rho_{1}, \xi \bar{\rho}\right)$. Such a diagram automorphism can be obtained by multiplying the "point-line involution" $\sigma$ (defined in section 3.1) with a diagram automorphism that permutes the points, since $Q$ acts transitively on the points. So there is a diagram automorphism that takes $\left(\bar{\rho}, q, \sigma_{1}\right)$ to $\left(\xi \bar{\rho}, \xi q, \xi \sigma_{2}\right)$.

For $k=1, \cdots, 6$, let $\Delta_{k}^{\prime}=\operatorname{Conv}\left(q^{\prime}, z_{k-1}, z_{k}\right)$. For $k=1,2,3$, let $\Delta_{k}^{\prime \prime}=$ $\operatorname{Conv}\left(q^{\prime}, z_{2 k-1}^{-}, z_{2 k-1}^{+}\right)$. So

$$
\bigcup_{x \in \sigma_{1}^{\prime}} \operatorname{Conv}\left(q^{\prime}, x\right) \subseteq\left(\bigcup_{k=1}^{3} \Delta_{k}^{\prime \prime}\right) \cup\left(\bigcup_{k=1}^{6} \Delta_{k}^{\prime} \backslash\left\{z_{1}, z_{3}, z_{5}\right\}\right) .
$$

In Table 2, we have recorded some inner products that we are going to need. Looking at the table we make the following observations:

(1) The numbers $c_{j}^{0}$ are always non-zero. In each row, at most one entry is zero.

(2) For all $j$ and $k$, we have $\operatorname{Re}\left(\xi c_{j}^{k}\right) \geq 0$.

(3) For each $k$, there is an open half plane $P_{k} \subseteq \mathbb{C}$ such that $\left\{c_{j}^{0}, \cdots, c_{j}^{6}\right\} \subseteq$ $P_{k} \cup\{0\}$.

Let $s_{1}, s_{2}, s_{3} \in \mathbb{R}$ such that $s_{i} \geq 0$ and $s_{1}+s_{2}+s_{3}=1$. Let $k \in\{1, \cdots, 6\}$ and let $w_{k}=s_{1} q^{\prime}+s_{2} z_{k-1}+s_{3} z_{k} \in \Delta_{k}^{\prime}$. If $\left\langle w_{k}, z_{j}\right\rangle=0$, then

$$
\left\langle w_{k}, r_{j}\right\rangle /|\bar{\rho}|^{2}=s_{1} \epsilon c_{j}^{0}+s_{2} c_{j}^{k-1}+s_{3} c_{j}^{k}=0,
$$

where $c_{j}^{k}$ are given in Table2. The three numbers $\left\{\epsilon c_{j}^{0}, c_{j}^{k-1}, c_{j}^{k}\right\}$ belong to $P_{k} \cup\{0\}$ for some open half plane $P_{k}$. So a convex combination of these three numbers is zero only when $c_{j}^{k-1}=0$ and $\left(s_{1}, s_{2}, s_{3}\right)=(0,1,0)$ or $c_{j}^{k}=0$ and $\left(s_{1}, s_{2}, s_{3}\right)=(0,0,1)$. It follows that equation (16) holds if and only if the pair $\left(w_{k}, r_{j}\right)$ is equal to $\left(z_{1}, r_{1}\right)$ or $\left(z_{3}, r_{3}\right)$ or $\left(z_{5}, r_{2}\right)$. So the only intersection of $\bigcup_{k=0}^{6} \Delta_{k}^{\prime}$ with $\bigcup_{j=1}^{4} r_{j}^{\perp}$ is at the points $\left\{z_{1}, z_{3}, z_{5}\right\}$. 
Now let $k \in\{1,2,3\}$ and let $w_{k}=s_{1} q^{\prime}+s_{2} z_{2 k-1}^{-}+s_{3} z_{2 k-1}^{+} \in \Delta_{k}^{\prime \prime}$. Then

$$
\left\langle w_{k}, r_{j}\right\rangle /|\bar{\rho}|^{2}=s_{1} \epsilon c_{j}^{0}+s_{2}\left(c_{j}^{2 k-1}+\epsilon c_{j}^{2 k-2}\right)+s_{3}\left(c_{j}^{2 k-1}+\epsilon c_{j}^{2 k}\right) .
$$

Note that the three numbers $\left\{\epsilon c_{j}^{0}, c_{j}^{2 k-1}+\epsilon c_{j}^{2 k-2}, c_{j}^{2 k-1}+\epsilon c_{j}^{2 k}\right\}$ always belong to some open half plane $P_{k}$. So a convex combination of these cannot be zero; that is, $\bigcup_{k=1}^{3} \Delta_{k}^{\prime \prime}$ does not intersect $\bigcup_{j=1}^{4} r_{j}^{\perp}$. When $\phi_{1}$ and $\phi_{2}$ commute, the calculations are much easier and are omitted.

\section{The FIXED POINTS OF DIAGRAM AUTOMORPHISMS}

Recall from section 3 that the diagram automorphisms $\operatorname{PGL}\left(3, \mathbb{F}_{3}\right)$ pointwise fix a 2 dimensional primitive sub-lattice $F$ spanned by $w_{\mathcal{P}}$ and $w_{\mathcal{L}}$. Let $z_{0}=w_{\mathcal{P}}+\theta w_{\mathcal{L}}$. Let $\Gamma_{F}=\{g \in \mathbb{P} \operatorname{Aut}(L): g(F)=F\}$. The aim of this section is to prove the following theorem.

Theorem 5.1. (a) There is an isometry $\beta: \mathbb{P}_{+}\left(F^{\mathbb{C}}\right) \rightarrow \mathcal{H}^{2}$ such that $\beta(\bar{\rho})=i$, $\beta\left(w_{\mathcal{P}}\right)=p, \beta\left(w_{\mathcal{L}}\right)=-p^{-1}$ and $\beta\left(z_{0}\right)=0$. (See Figure 4 .)

(b) The isometry $\beta$ induces a group homomorphism $c_{\beta}: \Gamma_{F} \rightarrow \mathrm{PSL}_{2}(\mathbb{R})$, given by $c_{\beta}(h)=\beta \circ h \circ \beta^{-1}$. The image of $c_{\beta}$ contains the congruence subgroup $\Gamma(13) \subseteq$ $\mathrm{PSL}_{2}(\mathbb{Z})$. In fact

$$
c_{\beta}\left(\Gamma_{F}\right) \cap \mathrm{PSL}_{2}(\mathbb{Z})=\nu^{-1} \Gamma_{0}(13) \nu
$$

where $\nu=\left(\begin{array}{cc}0 & 1 \\ -1 & 5\end{array}\right)$. Further, $c_{\beta}(\sigma)=S=\left(\begin{array}{cc}0 & -1 \\ 1 & 0\end{array}\right)$, where $\sigma \in \operatorname{Aut}(F)$ is the involution that corresponds to interchanging the points and lines of $\mathbb{P}^{2}\left(\mathbb{F}_{3}\right)$.

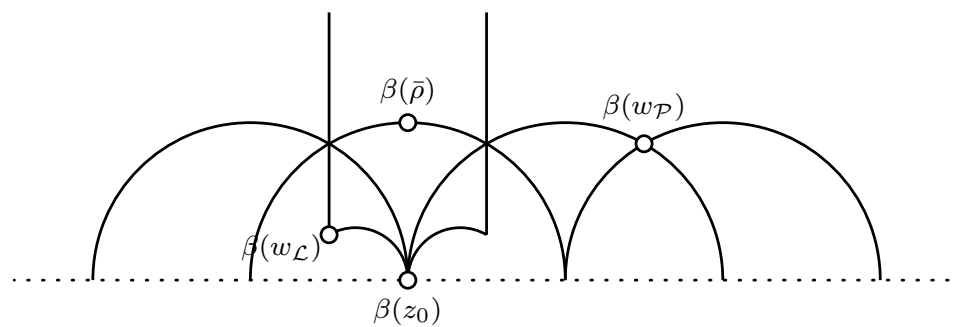

FIGURE 4

Remark 5.2. The stabilizer in $\operatorname{Aut}(L)$ of the norm zero vector $z_{0}$ contains $\operatorname{PGL}\left(3, \mathbb{F}_{3}\right)$. Theorem 4 of [3] implies that $z_{0}$ is a "cusp of Leech type"; that is, $z_{0}^{\perp} / z_{0}$ is isomorphic to the complex Leech lattice.

Remark 5.3. Let $\Phi_{L}$ be the set of roots of $L$ and $L_{+}^{\mathbb{C}}$ denote the set of vectors of $L^{\mathbb{C}}$ having positive norm. Consider $E_{m}: L_{+}^{\mathbb{C}} \rightarrow \mathbb{C}$, defined by

$$
E_{m}(z)=\sum_{r \in \Phi_{L}}\langle r, z\rangle^{-6 m}
$$

Fix $z \in L_{+}^{\mathbb{C}}$. The number of roots $r$ such that $|\langle r, z\rangle| \leq N$ grows as a polynomial in $N$. So the infinite sum above defines a non-constant meromorphic function if $m$ is large enough. The functions $E_{m}$ are invariant under $\operatorname{Aut}(L)$ and have poles exactly along the mirrors of the reflection group $R(L)$. The lattice $F$ is not contained in any mirror as there are no mirrors passing through $\bar{\rho} \in F^{\mathbb{C}}$. So the restriction of 
$E_{m}$ to $F_{+}^{\mathbb{C}}$ is a non-constant meromorphic function invariant under $\Gamma_{F}$. Theorem 5.1 shows that $\Gamma_{F}$ is commensurable with $\operatorname{PSL}_{2}(\mathbb{Z})$. So the restriction of $E_{m}$ to $F_{+}^{\mathbb{C}}$ is an ordinary meromorphic modular form of level 13 .

Starting with modular forms of singular weight with poles at cusps, one can construct meromorphic automorphic forms of type $U(1, n)$ by first taking Borcherds singular theta lift to get an automorphic form of type $O(2,2 n)$ and then restricting to the Hermitian symmetric space of $U(1, n)$ (see Theorem 7.1 of [1] and [7]). In our example, these Borcherds forms will be automorphic with respect to some finite index subgroup of $\operatorname{Aut}(L)$ and have their divisors along the mirrors, just like the functions $E_{m}(z)$. It will be interesting to understand how the restriction to $F_{+}^{\mathbb{C}}$ is related to the inverse of the Borcherds lift.

It follows from general considerations that $c_{\beta}\left(\Gamma_{F}\right)$ is commensurable with $\mathrm{PSL}_{2}(\mathbb{Z})$. (This was explained to me by Stephen Kudla.) But we want to calculate $c_{\beta}\left(\Gamma_{F}\right) \cap \mathrm{PSL}_{2}(\mathbb{Z})$ precisely, so we need to understand when an automorphism of $F$ extends to an automorphism of $L$. For this, we need the following lemma. We urge the reader to recall, at this point, the construction of $L$ from the diagram $D$ given in section 3 and equations (2), (4) and (5).

Lemma 5.4. Fix any $x_{0} \in \mathcal{P}$ and $l_{0} \in \mathcal{L}$. Let $\bar{L}=L /\left(F \oplus F^{\perp}\right)$. Let

$$
13 \bar{x}=\Sigma_{\mathcal{P}}=\sum_{x \in \mathcal{P}} x .
$$

Let $\pi_{F}: L^{\mathbb{C}} \rightarrow F^{\mathbb{C}}$ and $\pi_{F^{\perp}}: L^{\mathbb{C}} \rightarrow\left(F^{\perp}\right)^{\mathbb{C}}$ be the orthogonal projections.

(a) The lattice $F^{\perp}$ is spanned by the vectors $\left\{\left(x-x_{0}\right): x \in \mathcal{P}\right\} \cup\left\{\left(l-l_{0}\right): l \in \mathcal{L}\right\}$.

(b) One has $\Sigma_{\mathcal{P}}=4 w_{\mathcal{P}}-\bar{p} w_{\mathcal{L}} \in F$ and $\left(13 x_{0}-\Sigma_{\mathcal{P}}\right) \in F^{\perp}$. So $\pi_{F}\left(x_{0}\right)=\bar{x}$ and $\pi_{F^{\perp}}\left(x_{0}\right)=x_{0}-\bar{x}$.

(c) One has an isomorphism $\mathcal{E} / 13 \mathcal{E} \simeq \bar{L}$ obtained by sending 1 to the image of $x_{0}\left(\right.$ or $\left.l_{0}\right)$.

(d) Let $i_{F}: \bar{L} \rightarrow \mathrm{D}(F)=F^{\prime} / F$ be the injection given by $u \mapsto \pi_{F}(u) \bmod F$. Similarly define $i_{F^{\perp}}: \bar{L} \hookrightarrow \mathrm{D}\left(F^{\perp}\right)$. It follows from parts (b) and (c) that $i_{F}(\bar{L})$ is generated by $\bar{x}$ and $i_{F^{\perp}}(\bar{L})$ is generated by $\left(x_{0}-\bar{x}\right)$. The quotients $\mathrm{D}(F) / i_{F}(\bar{L})$ and $\mathrm{D}\left(F^{\perp}\right) / i_{F^{\perp}}(\bar{L})$ are 3 -groups.

(e) Given $g_{1} \in \operatorname{Aut}(F)$ and $g_{2} \in \operatorname{Aut}\left(F^{\perp}\right)$, one can extend $\left(g_{1} \times g_{2}\right)$ to an automorphism of $L$ if and only if

$$
i_{F}^{-1} \circ g_{1} \circ i_{F}=i_{F^{\perp}}^{-1} \circ g_{2} \circ i_{F^{\perp}} .
$$

(In other words, $g_{1}$ and $g_{2}$ act on the image of $\bar{L}$ in the same way.)

(f) The automorphism $\sigma$ acts on $i_{F^{\perp}}(\bar{L}) \subseteq \mathrm{D}\left(F^{\perp}\right)$ as multiplication by $3 \bar{p}$.

Proof. (a) Let $\tilde{F}=\operatorname{span}\left\{x-x_{0}, l-l_{0}: x \in \mathcal{P}, l \in \mathcal{L}\right\}$. Equation (5) implies $\tilde{F} \subseteq F^{\perp}$. Since $L$ is spanned by $\mathcal{P} \cup \mathcal{L}$, it is also spanned by $\tilde{F}$ together with $x_{0}$ and $l_{0}$. Given $w \in F^{\perp}$, we can write $w=\alpha_{1} x_{0}+\alpha_{2} l_{0}+\tilde{w}$, where $\tilde{w} \in \tilde{F}$. Taking the inner product with $w_{\mathcal{P}}$ and $w_{\mathcal{L}}$, one finds that $\alpha_{1}=\alpha_{2}=0$, so $w \in \tilde{F}$. This proves part (a).

(b) For each of the four lines $l$ passing through $x_{0}$, we have $w_{\mathcal{P}}=\bar{p} l+\sum_{x \in l} x$ (cf. equation (4)). Adding these together we get

$$
4 w_{\mathcal{P}}=\bar{p} \sum_{x_{0} \in l} l+3 x_{0}+\Sigma_{\mathcal{P}}=\bar{p} w_{\mathcal{L}}+\Sigma_{\mathcal{P}} .
$$

So $\Sigma_{\mathcal{P}} \in F$. Clearly $13 x_{0}-\Sigma_{\mathcal{P}}=\sum_{x \in \mathcal{P}}\left(x_{0}-x\right) \in F^{\perp}$. 
(c) Note that $\bar{p} l_{0}+4 x_{0}=w_{\mathcal{P}}+\sum_{x \in l_{0}}\left(x_{0}-x\right) \in F \oplus F^{\perp}$. Similarly $p x_{0}+4 l_{0} \in$ $F \oplus F^{\perp}$. So

$$
l_{0}-3 p x_{0}=\left(p x_{0}+4 l_{0}\right)-p\left(\bar{p} l_{0}+4 x_{0}\right) \in F \oplus F^{\perp} .
$$

So $F \oplus F^{\perp}$ together with $x_{0}$ generates $L$. Part (b) implies that $13 x_{0} \in F \oplus F^{\perp}$. Conversely suppose $\alpha x_{0} \in F \oplus F^{\perp}$. Choose $u, v \in \mathcal{E}$ such that $\left(\alpha x_{0}-u w_{\mathcal{P}}-\right.$ $\left.v w_{\mathcal{L}}\right) \in F^{\perp}$. Taking the inner product with $w_{\mathcal{L}}$ and $w_{\mathcal{P}}$ yields $\alpha \bar{p}=4 \bar{p} u+3 v$ and $0=3 u+4 p v$. So

$$
-13 p v=p(4 \bar{p} u+3 v)-4(3 u+4 p v)=3 \alpha ;
$$

that is, $\alpha$ is a multiple of 13 .

(d) By calculating discriminants we find that $|\mathrm{D}(F)|=13^{2} .3^{2}$ and $\left|\mathrm{D}\left(F^{\perp}\right)\right|=$ $13^{2} .3^{12}$. Since $|\bar{L}|=13^{2}$, it follows that $\mathrm{D}(F) / i_{F}(\bar{L})$ and $\mathrm{D}\left(F^{\perp}\right) / i_{F^{\perp}}(\bar{L})$ are 3 groups.

(e) Note that $i_{F}(\bar{L})$ is generated by $\pi_{F}\left(x_{0}\right)=\bar{x}$ and $i_{F^{\perp}}(\bar{L})$ is generated by $\pi_{F^{\perp}}\left(x_{0}\right)=x_{0}-\bar{x}$. From part (d) and the Chinese Remainder Theorem we find that $\mathrm{D}(F)$ is the direct product of $i_{F}(\bar{L})$ and a 3-group. So $g_{1}$ acts on $i_{F}(\bar{L})$ as multiplication by some $\lambda \in(\mathcal{E} / 13 \mathcal{E})^{*}$ (since $\bar{L}$ is a cyclic $\mathcal{E}$-module and $g_{1}$ is $\mathcal{E}$ linear). Similarly, as $\mathrm{D}\left(F^{\perp}\right)$ is the product of $i_{F^{\perp}}(\bar{L})$ and a 3-group, $g_{2}$ acts on $i_{F^{\perp}}(\bar{L})$ as multiplication by some $\lambda^{\prime}$. The condition $i_{F}^{-1} \circ g_{1} \circ i_{F}=i_{F^{\perp}}^{-1} \circ g_{2} \circ i_{F^{\perp}}$ is equivalent to $\lambda=\lambda^{\prime}$. If this condition is satisfied, we have

$\left(g_{1} \times g_{2}\right)\left(x_{0}\right)=g_{1}(\bar{x})+g_{2}\left(x_{0}-\bar{x}\right) \equiv \lambda \bar{x}+\lambda\left(x_{0}-\bar{x}\right) \bmod F \oplus F^{\perp} \equiv \lambda x_{0} \bmod F \oplus F^{\perp}$, which implies that $\left(g_{1} \times g_{2}\right)\left(x_{0}\right) \in L$. The converse is also clear.

(f) Assume that $\sigma\left(l_{0}\right)=x_{0}$. Let $\bar{l}=\sum_{l \in \mathcal{L}} l / 13$. Then $\pi_{F}\left(l_{0}\right)=\bar{l}$. We have seen in equation (18) that $v=l_{0}-3 p x_{0} \in F \oplus F^{\perp}$. So

$$
v-\pi_{F}(v)=\left(l_{0}-3 p x_{0}\right)-(\bar{l}-3 p \bar{x}) \in F^{\perp},
$$

that is, $\left(l_{0}-\bar{l}\right) \equiv 3 p\left(x_{0}-\bar{x}\right) \bmod F^{\perp}$. It follows that

$$
\sigma\left(x_{0}-\bar{x}\right)=-\omega\left(l_{0}-\bar{l}\right) \equiv-\omega(3 p)\left(x_{0}-\bar{x}\right) \bmod F^{\perp} .
$$

Definition 5.5 (of the isometry $\beta$ ). Note that $\left\langle w_{\mathcal{P}}, \xi w_{\mathcal{L}}\right\rangle=4 \sqrt{3} \in \mathbb{R}$. We let

$$
y_{ \pm}=2^{-\frac{1}{2}} 3^{-\frac{1}{4}}(p \pm i)^{-1}\left(w_{\mathcal{P}} \pm \xi w_{\mathcal{L}}\right) .
$$

Then $\left\{y_{+}, y_{-}\right\}$forms a basis for the vector space $F^{\mathbb{C}}$ and we have

$$
\left|y_{+}\right|^{2}=-\left|y_{-}\right|^{2}=1, \quad\left\langle y_{+}, y_{-}\right\rangle=0 \text {. }
$$

So $\beta^{\prime}: \mathbb{P}_{+}\left(F^{\mathbb{C}}\right) \rightarrow B^{1}(\mathbb{C})$ given by $\beta^{\prime}\left[u y_{+}+v y_{-}\right]=\frac{v}{u}$ is an isometry. Let $C(w)=$ $i\left(\frac{1+w}{1-w}\right)$ be the Cayley isomorphism from the disc to the upper half plane. Define

$$
\beta=C \circ \beta^{\prime}: P_{+}\left(F^{\mathbb{C}}\right) \rightarrow \mathcal{H}^{2} .
$$

The map $\beta$ is an isometry since both $\beta^{\prime}$ and $C$ are. After some simplification, one obtains

$$
\beta\left[a w_{\mathcal{P}}+b w_{\mathcal{L}}\right]=\frac{p a+\omega b}{a+\bar{p} b} \quad \text { and } \quad \beta^{-1}(\tau)=\left[(1+p \tau) w_{\mathcal{P}}+\omega^{2}(\tau-p) w_{\mathcal{L}}\right] .
$$


Notation. For the rest of this section, we shall represent $z=u w_{\mathcal{P}}+v w_{\mathcal{L}} \in F^{\mathbb{C}}$ as a column vector $z=\left(\begin{array}{l}u \\ v\end{array}\right)$. Accordingly, the isometry $\beta$ will be represented by the $\operatorname{matrix}\left(\begin{array}{cc}p & \omega \\ 1 & \bar{p}\end{array}\right)$.

Lemma 5.6. (a) Let $J_{F}=\left(\begin{array}{ll}\left\langle w_{\mathcal{P}}, w_{\mathcal{P}}\right\rangle & \left\langle w_{\mathcal{P}}, w_{\mathcal{L}}\right\rangle \\ \left\langle w_{\mathcal{L}}, w_{\mathcal{P}}\right\rangle & \left\langle w_{\mathcal{L}}, w_{\mathcal{L}}\right\rangle\end{array}\right)=\left(\begin{array}{cc}3 & 4 p \\ 4 \bar{p} & 3\end{array}\right)$ (see equation (51)). Then

$$
J_{F}=\bar{\theta} \beta^{*} S \beta .
$$

Let $\beta z=\left(\begin{array}{c}\tau_{1} \\ \tau_{2}\end{array}\right), \beta z^{\prime}=\left(\begin{array}{c}\tau_{1}^{\prime} \\ \tau_{2}^{\prime}\end{array}\right)$. Then $\left\langle z, z^{\prime}\right\rangle=\bar{\theta}\left(\tau_{1}^{\prime} \bar{\tau}_{2}-\tau_{2}^{\prime} \bar{\tau}_{1}\right)$. In particular $|z|^{2}=$ $2 \sqrt{3} \operatorname{Im}\left(\tau_{1} \bar{\tau}_{2}\right)$.

(b) Let $\nu=\left(\begin{array}{cc}0 & 1 \\ -1 & 5\end{array}\right)$. Given $g=\left(\begin{array}{ll}a & b \\ c & d\end{array}\right) \in \mathrm{SL}_{2}(\mathbb{Z})$, let $g_{1}=\beta^{-1} g \beta$. Then $\left(z \mapsto g_{1} z\right)$ is an isometry of the Hermitian vector space $F^{\mathbb{C}}$. Further, $g_{1} \in \operatorname{Aut}(F)$ if and only if

$$
g \in \nu^{-1} \Gamma_{0}(13) \nu=\left\{\left(\begin{array}{ll}
a & b \\
c & d
\end{array}\right) \in \mathrm{SL}_{2}(\mathbb{Z}): 5(a-d)+(b+c) \equiv 0 \bmod 13\right\}
$$

Proof. (a) The equation (20) is verified by multiplying matrices. Given $z, z^{\prime} \in F^{\mathbb{C}}$, one has

$$
\left\langle z, z^{\prime}\right\rangle=z^{*} J_{F} z^{\prime}=\bar{\theta} z^{*} \beta^{*} S \beta z^{\prime}=\bar{\theta}\left(\bar{\tau}_{1} \bar{\tau}_{2}\right) S\left(\begin{array}{l}
\tau_{1}^{\prime} \\
\tau_{2}^{\prime}
\end{array}\right)=\bar{\theta}\left(\tau_{1}^{\prime} \bar{\tau}_{2}-\tau_{2}^{\prime} \bar{\tau}_{1}\right) .
$$

(b) To show that $g_{1}$ is an isometry we calculate as follows:

$$
\begin{aligned}
\left\langle g_{1} z_{1}, g_{1} z_{2}\right\rangle & =z_{1}^{*} \beta^{*} g^{*}\left(\beta^{-1}\right)^{*} J_{F} \beta^{-1} g \beta z_{2}=\bar{\theta} z_{1}^{*} \beta^{*} g^{*} S g \beta z_{2} \\
& =\bar{\theta} z_{1}^{*} \beta^{*} S \beta z_{2}=z_{1}^{*} J_{F} z_{2}=\left\langle z_{1}, z_{2}\right\rangle .
\end{aligned}
$$

The second equality uses (20), and the third one follows from $g^{\text {tr }} S g=S$ for all $g \in \mathrm{Sp}_{2}(\mathbb{Z})$.

Let $p_{1}=\operatorname{det}(\beta)=3-\omega$. Then $p_{1} \bar{p}_{1}=13$. Let $a-d=s$ and $b+c=t$. We find that

$$
g_{1}=\frac{1}{p_{1}}\left(\begin{array}{cc}
p_{1} a+\omega s+\bar{p} t & -\omega p_{1} b-\bar{\omega} p s-\bar{\omega} t \\
-\bar{\omega} p_{1} c-p s-t & p_{1} d-\omega s-\bar{p} t
\end{array}\right) .
$$

Note that $\omega s+\bar{p} t \equiv \bar{p}(p s+t) \bmod p_{1}$. So the entries of the matrix $g_{1}$ belong to $\mathcal{E}$ if and only if $p_{1}$ divides $(p s+t)$ in $\mathcal{E}$. This is equivalent to the congruence condition $5 s+t \equiv 0 \bmod 13 \mathbb{Z}$. On the other hand, one checks by direct congruence calculation that

$$
\nu^{-1} \Gamma_{0}(13) \nu=\left\{\left(\begin{array}{ll}
a & b \\
c & d
\end{array}\right) \in \mathrm{SL}_{2}(\mathbb{Z}): 5(a-d)+(b+c) \equiv 0 \bmod 13\right\} .
$$

Proof of Theorem [5.1. The isometry $\beta$ has been defined in Definition [5.5. The claims $\beta(\bar{\rho})=i, \beta\left(w_{\mathcal{P}}\right)=-\beta\left(w_{\mathcal{L}}\right)^{-1}=p$ and $\beta\left(w_{\mathcal{P}}+\theta w_{\mathcal{L}}\right)=0$ are easily checked using (19). The automorphism $\sigma$ interchanges $w_{\mathcal{P}}=\beta^{-1}(p)$ and $w_{\mathcal{L}}=\beta^{-1}\left(-p^{-1}\right)$ and fixes $\bar{\rho}=\beta^{-1}(i)$. So $c_{\beta}(\sigma)=S$.

Suppose $g \in \mathrm{SL}_{2}(\mathbb{Z})$ such that $\nu g \nu^{-1} \in \Gamma_{0}(13)$. Lemma 5.6(b) shows that $g_{1}=\beta^{-1} g \beta$ is an isometry of the lattice $F$. Since $i_{F}(\bar{L})$ is generated by $\bar{x}$ (see Lemma $5.4(\mathrm{~d}))$, there exists $\lambda \in(\mathcal{E} / 13 \mathcal{E})^{*}$ such that $g_{1}(\bar{x}) \equiv \lambda \bar{x} \bmod F$. From 
Lemma 5.4(e) we find that $g_{1}$ can be extended to an automorphism of $L$ if there exists an automorphism of $F^{\perp}$ that acts on $i_{F^{\perp}}(\bar{L})$ as multiplication by $\lambda$. In particular, Lemma 5.4(f) implies that $g_{1}$ can be extended to $L$ if $\lambda$ belongs to the multiplicative subgroup of $(\mathcal{E} / 13 \mathcal{E})^{*}$ generated by $3 \bar{p}$. We check this by a direct calculation, sketched below. The congruences somehow work out exactly as we need. Looking at this arithmetic it seems that a computation-free, conceptual argument must exist.

Recall from Lemma 5.4 (b) that $\pi_{F}(x)=\Sigma_{\mathcal{P}} / 13$, where $\Sigma_{\mathcal{P}}=4 w_{\mathcal{P}}-\bar{p} w_{\mathcal{L}} \in F$. So in our matrix notation, $\Sigma_{\mathcal{P}}=\left({ }_{-\bar{p}}^{4}\right)$. Observe that $\beta\left(\begin{array}{c}4 \\ -\bar{p}\end{array}\right)=\operatorname{det}(\beta)\left(\begin{array}{c}p \\ -\bar{\omega}\end{array}\right)$. Lemma 5.6 implies that

$$
g \equiv\left(\begin{array}{cc}
s+d & b \\
-5 s-b & d
\end{array}\right) \bmod 13
$$

for some $s \in \mathbb{Z}$. Using this, one checks that

$$
g_{1}\left(\begin{array}{c}
4 \\
-\bar{p}
\end{array}\right)=\beta^{-1} g \beta\left(\begin{array}{c}
4 \\
-\bar{p}
\end{array}\right) \equiv \lambda\left(\begin{array}{c}
4 \\
-\bar{p}
\end{array}\right) \bmod 13,
$$

where $\lambda=[8 s(4+3 \omega)+d+3 p \bar{\omega} b] \bmod 13 \mathcal{E}$. In other words, $g_{1}(\bar{x}) \equiv \lambda \bar{x} \bmod F$. Recall the prime factorization $13=p_{1} \bar{p}_{1}$, where $p_{1}=3-\omega$. By the Chinese Remainder Theorem, we have an isomorphism

$$
\varphi: \mathcal{E} / 13 \mathcal{E} \rightarrow \mathcal{E} / p_{1} \mathcal{E} \times \mathcal{E} / \bar{p}_{1} \mathcal{E} \simeq \mathbb{F}_{13} \times \mathbb{F}_{13} \text {, given by } x \mapsto\left(x \bmod p_{1}, x \bmod \bar{p}_{1}\right) .
$$

Now, using $\varphi(\omega)=(3,9)$ and $\varphi(\bar{\omega})=(9,3)$, it follows that

$$
\varphi(3 \bar{p})=(7,2)=\left(2^{-1}, 2\right) \in \mathbb{F}_{13} \times \mathbb{F}_{13} \text { and } \varphi(\lambda)=(d+5 b, s+d+8 b) .
$$

Since 2 is a generator of $\mathbb{F}_{13}^{*}$, it follows that $\lambda$ belongs to the multiplicative group generated by $3 \bar{p}$ if and only if $\varphi(\lambda)$ has the form $(u, v)$ with $u . v=1$. To finish the proof, we note that

$$
(d+5 b)(s+d+8 b) \equiv s(d+5 b)+d^{2}+b^{2} \equiv \operatorname{det}(g) \equiv 1 \bmod 13 .
$$

Lemma 5.7. (a) Let $z \in F$ be a norm 3 vector. Then there exists $g_{z} \in \mathrm{SL}_{2}(\mathbb{Z})$ such that $\beta(z)=g_{z}(\omega)$.

(b) Let $r \in F$ be a vector of norm -3. Let $z \in F \cap r^{\perp}$ be a primitive non-zero vector. Then there exists $g_{z} \in \mathrm{SL}_{2}(\mathbb{Z})$ such that $\beta(z)=g_{z}(\omega)$.

Proof. (a) Let $z \in F$. Let us write $\beta . z=\left(\begin{array}{l}s \\ t\end{array}\right)=\left(\begin{array}{c}s_{1} \omega+s_{2} \\ t_{1} \omega+t_{2}\end{array}\right)$ with $s_{j}, t_{j} \in \mathbb{Z}$. Part (a) of Lemma 5.6 implies that $|z|^{2}=3$ if and only if $\sqrt{3}=2 \operatorname{Im}(\bar{t} s)$, which simplifies to $s_{1} t_{2}-s_{2} t_{1}=1$. So we can take $g_{z}=\left(\begin{array}{ll}s_{1} & s_{2} \\ t_{1} & t_{2}\end{array}\right)$.

(b) Let $\beta . r=\left(\begin{array}{l}s \\ t\end{array}\right)$. Now $|r|^{2}=-3$ implies $2 \operatorname{Im}(\bar{t} s)=-\sqrt{3}$. So $2 \operatorname{Im}(\bar{s} \bar{t})=\sqrt{3}$. As in part (a), this implies $\bar{s} / \bar{t} \in \mathrm{PSL}_{2}(\mathbb{Z}) \omega$. Now let $\beta . z=\left(\begin{array}{l}u \\ v\end{array}\right)$. One has $0=$ $\langle z, r\rangle=\bar{\theta}(-\bar{s} v+\bar{t} u)$. It follows that $\beta(z)=u / v=\bar{s} / \bar{t} \in \mathrm{PSL}_{2}(\mathbb{Z}) \omega$.

Remark 5.8. The stabilizer of $\omega \in \mathcal{H}^{2}$ in $\operatorname{PSL}_{2}(\mathbb{Z})$ is the $\mathbb{Z} / 3 \mathbb{Z}$ generated by $S T$. If $z \in F$ has norm 3 , then the $120^{\circ}$ rotation around $z \in \mathbb{P}_{+}(F)$ belongs to $\Gamma_{F}$. If $r \in F$ is a vector of norm -3 and $z$ is a primitive vector in $F \cap r^{\perp}$, then the restriction of $\phi_{r}^{\omega}$ to $\mathbb{P}_{+}(F)$ is an element of $\Gamma_{F}$, which is again a $120^{\circ}$ rotation around $z$. Lemma 5.7 implies that the images of these rotations under $c_{\beta}$ are $\mathrm{PSL}_{2}(\mathbb{Z})$ conjugates of $S T$ or $(S T)^{-1}$. 


\section{ACKNOWLEDGMENTS}

The author wants to thank Professor Jon Alperin, Professor Richard Borcherds, Professor John Conway, Professor Benson Farb, Professor George Glauberman, Professor Stephen Kudla and Professor John Mckay for encouragement and useful conversations. The author is also very grateful to the referee for pointing out several mistakes in the preprint and for suggesting many improvements. Most of all, the author wants to thank Professor Daniel Allcock for generously sharing his insights and ideas on this project.

\section{REFERENCES}

[1] D. Allcock, The Leech lattice and complex hyperbolic reflections, Invent. Math. 140 (2000), no. 2, 283-301, DOI 10.1007/s002220050363. MR:1756997 (2002b:11091)

[2] D. Allcock, A monstrous proposal, Groups and symmetries, CRM Proc. Lecture Notes, vol. 47, Amer. Math. Soc., Providence, RI, 2009, pp. 17-24. MR 2500552 (2010k:20022)

[3] D. Allcock, On the $Y_{555}$ complex reflection group, J. Algebra 322 (2009), no. 5, 1454-1465, DOI 10.1016/j.jalgebra.2009.05.027. MR2543618(2010g:20068)

[4] D. Allcock and T. Basak, Geometric generators for braid-like groups, arXiv:1403:2401, (2014), to appear in Geometry and Topology.

[5] T. Basak, The complex Lorentzian Leech lattice and the Bimonster, J. Algebra 309 (2007), no. 1, 32-56, DOI 10.1016/j.jalgebra.2006.05.033. MR2301231(2008f:20140)

[6] T. Basak, Modular lattices from finite projective planes (English, with English and French summaries), J. Théor. Nombres Bordeaux 26 (2014), no. 2, 269-279. MR3320480

[7] R. E. Borcherds, Automorphic forms with singularities on Grassmannians, Invent. Math. 132 (1998), no. 3, 491-562, DOI 10.1007/s002220050232. MR:1625724 (99c:11049)

[8] E. Brieskorn and K. Saito, Artin-Gruppen und Coxeter-Gruppen (German), Invent. Math. 17 (1972), 245-271. MR0323910 (48 \#2263)

[9] J. H. Conway, S. P. Norton, and L. H. Soicher, The Bimonster, the group $Y_{555}$, and the projective plane of order 3, Computers in algebra (Chicago, IL, 1985), Lecture Notes in Pure and Appl. Math., vol. 111, Dekker, New York, 1988, pp. 27-50. MR.1060755 (92f:20018)

[10] J. H. Conway and A. D. Pritchard, Hyperbolic reflections for the Bimonster and $3 \mathrm{Fi}_{24}$, Groups, combinatorics \& geometry (Durham, 1990), London Math. Soc. Lecture Note Ser., vol. 165, Cambridge Univ. Press, Cambridge, 1992, pp. 24-45, DOI 10.1017/CBO9780511629259.006. MR1200248 (94a:20033)

[11] J. H. Conway and C. S. Simons, 26 implies the Bimonster, J. Algebra 235 (2001), no. 2, 805-814, DOI 10.1006/jabr.2000.8494. MR.1805481(2001k:20028)

[12] J. H. Conway and N. J. A. Sloane, Sphere packings, lattices and groups, 3rd ed., with additional contributions by E. Bannai, R. E. Borcherds, J. Leech, S. P. Norton, A. M. Odlyzko, R. A. Parker, L. Queen and B. B. Venkov. Grundlehren der Mathematischen Wissenschaften [Fundamental Principles of Mathematical Sciences], vol. 290, Springer-Verlag, New York, 1999. MR 1662447 (2000b:11077)

[13] P. Deligne, Les immeubles des groupes de tresses généralisés (French), Invent. Math. 17 (1972), 273-302. MR0422673 (54 \#10659)

[14] W. M. Goldman, Complex hyperbolic geometry, Oxford Mathematical Monographs, The Clarendon Press, Oxford University Press, New York, 1999. MR,1695450 (2000g:32029)

[15] G. Heckman, The Allcock ball quotient, arXiv:1307.1339, (2013).

[16] G. Heckman and S. Reiken, Two Lorentzian lattices, arXiv:1412.2922, (2014).

[17] A. A. Ivanov, A geometric characterization of the Monster, Groups, combinatorics \& geometry (Durham, 1990), London Math. Soc. Lecture Note Ser., vol. 165, Cambridge Univ. Press, Cambridge, 1992, pp. 46-62, DOI 10.1017/CBO9780511629259.007. MR.1200249 (94c:20033)

[18] A. A. Ivanov, Y-groups via transitive extension, J. Algebra 218 (1999), no. 2, 412-435, DOI 10.1006/jabr.1999.7882. MR1705810 (2000i:20029)

[19] M. Liebeck and J. Saxl (eds.), Groups, combinatorics \& geometry, London Mathematical Society Lecture Note Series, vol. 165, Cambridge University Press, Cambridge, 1992. MR 1200244 (93g:20001) 
[20] E. Looijenga, Compactifications defined by arrangements. I. The ball quotient case, Duke Math. J. 118 (2003), no. 1, 151-187, DOI 10.1215/S0012-7094-03-11816-5. MR1978885 (2004i:14042a)

[21] S. P. Norton, Constructing the Monster, Groups, combinatorics \& geometry (Durham, 1990), London Math. Soc. Lecture Note Ser., vol. 165, Cambridge Univ. Press, Cambridge, 1992, pp. 63-76, DOI 10.1017/CBO9780511629259.008. MR.1200250 (94c:20034)

Department of Mathematics, Iowa State University, Ames, Iowa 50011

E-mail address: tathagat@iastate.edu

$U R L:$ http://orion.math.iastate.edu/tathagat 